Pacific Journal of Mathematics

COHOMOLOGY OVER BANACH CROSSED PRODUCTS.
PPLICATION TO BOUNDED DERIVATIONS AND CROSSED

GUY LOUPIAS 


\title{
COHOMOLOGY OVER BANACH CROSSED PRODUCTS. APPLICATION TO BOUNDED DERIVATIONS AND CROSSED HOMOMORPHISMS
}

\author{
GUY LOUPIAS
}

\begin{abstract}
The purpose of this work is to study the structure of bounded derivations and crossed homomorphisms of the Banach crossed product $\mathfrak{U}=L^{1}(G, A)$ of a Banach-*-algebra $A$ acted upon by a locally compact group $G$. As bounded derivations and crossed homomorphisms are related to 1-cocycles, we first define and study cohomology over $\mathfrak{A}$, generalizing cohomology over group algebras. Then, if $G$ is amenable and $A$ is a $C^{*}$-algebra, or the dual of a Banach space, we show that a bounded derivation (resp. a crossed homomorphism) on $\mathfrak{A}$ is equivalent to some couple of a bounded derivation (resp. a crossed homomorphism) from $A$ to $M_{1}(G, A)$ and a bounded measure on $A$ with value in the centralizers of $A$ (resp. an element of $\mathfrak{i}$ ).
\end{abstract}

1. Introduction. Crossed products of Banach algebras and locally compact groups are interesting objects from a mathematical point of view because they are generalizations of group algebras, from a physical point of view because they are useful tools in describing quantum dynamical systems. Hence it would be interesting to know the structure of their automorphisms and derivations. For a large class of automorphisms, the answer is given in [2]. In this paper, our aim is to begin the study of bounded derivations and crossed homomorphisms of Banach crossed products. For that purpose, cohomology techniques seem to be useful and this is the reason why we will begin with cohomology over Banach crossed products, a generalization of cohomology over group algebras worked out in [15].

Given a locally compact group $G$ acting on a Banach *-algebra $A, \mathfrak{A}=L^{1}(G, A)$ will be the Banach crossed product of these two objects. In paragraph 2 , we collect known results about centralizers on $A$ and vector measures, and define several module structures on them in paragraph 3. Paragraph 4 is devoted to the definition of cohomology over $\mathfrak{A}$, while paragraph 5 contains a Riesz representation theorem for the elements of the spaces introduced in the preceding paragraph. In paragraph 6 we extend the cohomology over $\mathfrak{A}$ to its centralizers. Finally paragraph 8 characterizes the structure of derivations and crossed homomorphisms, using the notion of vector means developed in paragraph 7 . 
2. Notations and preliminary results. In the sequel, $(A, G, \sigma)$ will denote a Banach dynamical system, that is to say the triplet of a (separable) Banach-*-algebra with norm $|\cdot|$ and (countable) approximate unit $\left\{e_{\alpha}\right\}_{\alpha \in I}$ contained in the unit ball, a second countable locally compact Hausdorff (hence Polish, i.e., second countable, metrizable and complete) group $G$ with Haar measure $d g$, and a representation $\sigma$ of $G$ into Aut $G$ (the group of continuous and isometric *-automorphisms of $A$ ), representation continuous in the sense that

$$
(a, g) \in A \times G \longrightarrow \sigma(g) a \in A
$$

is continuous.

Once $A$ is given, we call $M_{L}(A)$ (respectively $M_{R}(A), M(A)$ ) the space of left centralizers (resp. right centralizers, centralizers) on $A$. Let us recall [2], [16], [3] that $M_{L}(A)$ (resp. $M_{R}(A)$ ) is the algebra with unit of continuous linear maps $L$ (resp. $R$ ) on $A$ such that $L(a b)=L(a) b$ (resp. $R(a b)=a R(b))$ for any $a$ and $b$ in $A$, the product being defined by $L_{1} \cdot L_{2}=L_{1} \circ L_{2}$ (the composition of maps) (resp. $\left.R_{1} \cdot R_{2}=R_{2} \circ R_{1}\right) . \quad M(A)$ is the *-algebra with unit of couples $(L, R)$ of (automatically linear and continuous) maps on $A$ such that $a L(b)=R(a) b$ for any $a$ and $b$ in $A$, the product and *-operation being defined according to $\left(L_{1}, R_{1}\right) \cdot\left(L_{2}, R_{2}\right)=\left(L_{1} \cdot L_{2}, R_{1} \cdot R_{2}\right)=\left(L_{1} \circ L_{2}\right.$, $\left.R_{2} \circ R_{1}\right)$ and $(L, R)^{*}=\left(R^{\prime}, L^{\prime}\right)$ where $R^{\prime}(a)=R\left(a^{*}\right)^{*}$ and $L^{\prime}(a)=L\left(a^{*}\right)^{*}$. If $(L, R) \in M(A)$, then $L \in M_{L}(A)$ and $R \in M_{R}(A)$, and these algebras become Banach algebras under the operator norms:

$$
\|(L, R)\|=\|L\|=\|R\|=\lim _{\alpha}\left|L\left(e_{\alpha}\right)\right|=\lim _{\alpha}\left|R\left(e_{\alpha}\right)\right| .
$$

Through the correspondence $a \in A \rightarrow L_{a} \in M_{L}(A): L_{a} b=a b, b \in A$ (resp. $\left.a \in A \rightarrow R_{a} \in M_{R}(A): R_{a} b=b a, b \in A\right), A$ becomes a closed left ideal (resp. right ideal, *-ideal) of $M_{L}(A)$ (resp. $\left.M_{R}(A), M(A)\right)$ and $M(A)$ is the idealizer of $A$ in $M_{L}(A)$ (or $M_{R}(A)$ ). Moreover, $A$ is dense in $M_{L}(A)$ (resp. $M_{R}(A), M(A)$ ) for the strong (resp. strong, strict) topology, i.e., the topology defined by the set of semi-norms $\|L\|_{a}=$ $|L(a)|=|L \cdot a|, a \in A$ (resp. $\|R\|^{a}=|R(a)|=|a \cdot R| ;\|(L, R)\|_{a}=\|L\|_{a}$ and $\left.\|(L, R)\|^{a}=\|R\|^{a}\right)$. The formulas

$$
\left\{\begin{array}{l}
\sigma(g)(L \cdot a)=\sigma(g) L \cdot \sigma(g) a \\
\sigma(g)(a \cdot R)=\sigma(g) a \cdot \sigma(g) R \\
\sigma(g)(L, R)=(\sigma(g) L, \sigma(g) R
\end{array}\right.
$$

allow to extend $\sigma(g)$ as a continuous automorphism of $M_{L}(A), M_{R}(A)$ or $M(A)$.

If $A$ is a $C^{*}$-algebra, $M_{L}(A)$ (resp. $\left.M_{R}(A)\right)$ is isomorphic to the 
algebra $L M(A)$ (resp. $R M(A)$ ) of left (resp. right) multipliers on $A$, i.e., the subalgebra of the enveloping Von Neumann algebra $A^{\prime \prime}$ of elements $L \in A^{\prime \prime}$ (resp. $R \in A^{\prime \prime}$ ) such that $L a \in A$ (resp. $a R \in A$ ) and $M(A)=M_{L}(A) \cap M_{R}(A)$ is the idealizer of $A$ in $A^{\prime \prime}$. Moreover, if we now call $M_{L}\left(A, A^{\prime \prime}\right)$ (resp. $M_{R}\left(A, A^{\prime \prime}\right)$ ) the algebra of continuous linear maps $L$ (resp. $R$ ) from $A$ to $A^{\prime \prime}$ such that $L(a b)=L(a) b$ (resp. $R(a b)=a R(b)$ ), then it is possible to prove in the same way that $M_{L}\left(A, A^{\prime \prime}\right)=M_{R}\left(A, A^{\prime \prime}\right)=A^{\prime \prime}$. And here too, $\sigma(g)$ extends to $A^{\prime \prime}$ by bitransposition as a normal automorphism.

We will now denote by $\mathfrak{X}=\mathscr{C}_{0}(G, A)$ the Banach space of continuous functions from $G$ to $A$ "vanishing at infinity" with the uniform norm $\|h\|_{\infty}=\sup _{g \in G}|h(g)|, h \in \mathfrak{X}$ : it contains, as a dense set, the subspace $K(G, A)$ of continuous functions from $G$ to $A$ with compact support.

If $X=\mathscr{L}(E, F)$, the continuous operators from a Banach space $E$ to a Banach space $F$, with norms $\|\cdot\|_{E}$ and $\|\cdot\|_{F}$ respectively, $M_{1}(G, X)$ will be the Banach space of regular Borel measures $\mu$ on $G$ with bounded variation $|\mu|$ and norm $\|\mu\|_{1}=|\mu|(G)<\infty$. Let us recall [7] that if $B \in B(G)$, the ring of Borel sets in $G$, the variation $|\mu|$ of $\mu$ is the positive scalar measure on $G$ defined by

$$
|\mu|(B)=\sup \sum_{i}\left\|\mu\left(B_{i}\right)\right\|
$$

where the sup is over all (finite) families of mutually disjoint Borel sets $B_{i}$ contained in $B$. Then $\mu$ is said with finite variation if $|\mu|(B)<\infty$ for any relatively compact $B$ in $B(G)$ and with bounded variation if $|\mu|(G)<\infty$.

Let now $U$ be a linear mapping from $K(G, E)$ to $F$. In the usual way, we define

$$
\|U\|=\sup _{\|h\|_{\infty} \leqq 1}\|U(h)\|_{F}, \quad h \in K(G, E) .
$$

It is a norm and

$$
\|U(h)\|_{F} \leqq\|U\|\|h\|_{\infty}
$$

so that, if $\|U\|<\infty$, $U$ extends to $\mathscr{C}_{0}(G, E)$ by continuity. One can notice that $\|U\|$ can also be defined according to

$$
\|U\|=\sup \left\|\Sigma U\left(h_{i}\right)\right\|_{F}
$$

where the sup is over all finite families of functions $h_{i} \in K(G, E)$ such that support $h_{i} \cap$ support $h_{j}=\varnothing$ for any $i \neq j$ and $\left\|h_{i}\right\|_{\infty} \leqq 1$ (or equivalently $\left\|\Sigma_{i} h_{i}\right\|_{\infty} \leqq 1$ ).

In an analogous way, we can now define [2], [7] 


$$
\|U\|\left\|=\sup \sum_{i}\right\| U\left(h_{i}\right) \|_{F}
$$

where the sup is taken over the some families as in $\left(4^{\text {bis }}\right)$. It is a norm and

$$
\sum_{i}\left\|U\left(h_{i}\right)\right\|_{F} \leqq\|U\|\|\| \sum_{i} h_{i} \|_{\infty}
$$

for any finite family of functions $h_{i} \in K(G, E)$ with support $h_{i} \cap$ support $h_{j}=\varnothing$ for $i \neq j$, while

$$
\|U\| \leqq\|U\| ;\|U(h)\|_{F} \leqq\|U\|\|\| h \|_{\infty}
$$

so that, if ||$U \mid \|<\infty$, $U$ extends to $\mathscr{C}_{0}(G, E)$ by continuity.

It is now possible to prove the following theorem:

Theorem 1. Let $A$ be a Banach-*-algebra and $X=\mathscr{L}(E, F)$, where $E$ and $F$ are two Banach spaces.

(a) There exists a one-to-one linear correspondence between $M_{1}(G, X)$ and the Banach space of linear mappings $U$ from $\mathscr{C}_{0}(G, E)$ to $F$ such that ||$|U| \|<\infty$, given by

$$
\mu \longleftrightarrow U_{\mu}: U_{\mu^{\prime}}(h)=\langle\mu, h\rangle=\int d \mu(g) h(g), h \in \mathscr{C}_{0}(G, E), \mu \in M_{1}(G, X)
$$

with

$$
\text { | } \mid U_{\mu}\|\|=\|\mu\|_{1} .
$$

(b) This correspondence induces a one-to-one isometric correspondence between $M_{1}\left(G, M_{L}(A)\right.$ ) (resp. $M_{1}\left(G, M_{R}(A)\right.$ ) and the Banach space of $A$-right linear (resp. A-left linear) mappings $\cup$ from $\mathfrak{X}$ to $A$ such that $\|U\| \mid<\infty$.

(c) If $A$ is a $C^{*}$-algebra, this correspondence induces a one-toone isomorphic correspondence between $M_{1}\left(G, A^{\prime \prime}\right)$ and the Banach space of A-right linear (or A-left linear) mappings $U$ from $\mathfrak{X}$ to $A^{\prime \prime}$ such that $\|U \mid\|<\infty$.

Proof. (a) and (b) come from ([7], §19 no. 3, Theorem 2) and ([2], Theorem 3.9), while (c) can be proved in the same way as (b) thanks to

$$
M_{L}\left(A, A^{\prime \prime}\right)=M_{R}\left(A, A^{\prime \prime}\right)=A^{\prime \prime} .
$$

If we adopt the notations

$$
M_{1}\left(G, M_{L}(A)\right)=\mathfrak{X}_{L}^{*, A}, M_{1}\left(G, M_{R}(A)\right)=\mathfrak{X}_{R}^{*, A} ; M_{1}\left(G, A^{\prime \prime}\right)=\mathfrak{X}^{*, A^{\prime \prime}}
$$

(any time we use $A^{\prime \prime}$ without comment, we mean implicitely that $A$ is a $C^{*}$-algebra) we can write, for $\mu_{L} \in \mathfrak{X}_{L}^{*, A}, \mu_{R} \in \mathfrak{X}_{R}^{*, A}, \mu \in \mathfrak{X}^{*, A^{\prime \prime}}, h \in \mathfrak{X}$ 
$\left(12^{\text {bis }}\right)$

$$
\begin{aligned}
& \left\{\begin{array}{l}
\left\langle\mu_{L}, h\right\rangle \in A,\left\langle\mu_{L}, h a\right\rangle=\left\langle\mu_{L}, h\right\rangle a, a \in A \\
\langle\mu, h\rangle \in A^{\prime \prime},\langle\mu, h a\rangle=\langle\mu, h\rangle a
\end{array}\right. \\
& \left\{\begin{array}{l}
\left\langle h, \mu_{R}\right\rangle \in A,\left\langle a h, \mu_{R}\right\rangle=a\left\langle h, \mu_{R}\right\rangle, a \in A \\
\langle h, \mu\rangle \in A^{\prime \prime},\langle a h, \mu\rangle=a\langle h, \mu\rangle .
\end{array}\right.
\end{aligned}
$$

Given $g \in G$ and $\mu_{L}$ (resp. $\left.\mu_{R}, \mu\right)$ we define

$$
\begin{gathered}
{ }^{g} \mu_{L}(B)=\sigma(g) \mu_{L}(B)\left(\text { resp. }{ }^{g} \mu_{R}(B)=\sigma(g) \mu_{R}(B),\right. \\
\left.{ }^{g} \mu(B)=\sigma(g) \mu(B)\right), B \in B(G)
\end{gathered}
$$

or, in an equivalent way

$$
\left\{\begin{array}{l}
\left\langle{ }^{g} \mu_{L}, h\right\rangle=\sigma(g)\left\langle\mu_{L}, \sigma\left(g^{-1}\right) h\right\rangle\left(\mathrm{resp} .\left\langle h,{ }^{g} \mu_{R}\right\rangle=\sigma(g)\left\langle\sigma\left(g^{-1}\right) h, \mu_{R}\right\rangle,\right. \\
\left.\left\langle{ }^{g} \mu, h\right\rangle=\sigma(g)\left\langle\mu, \sigma\left(g^{-1}\right) h\right\rangle,\left\langle h,{ }^{g} \mu\right\rangle=\sigma(g)\left\langle\sigma\left(g^{-1}\right) h, \mu\right\rangle\right) .
\end{array}\right.
$$

Of course,

$$
\left\{\begin{array}{l}
\left|{ }_{\sigma} \mu_{L}\right|=\left|\mu_{L}\right|,\left|{ }^{g} \mu_{R}\right|=\left|\mu_{R}\right|,\left|{ }^{g} \mu\right|=|\mu| \\
\left\|\left.\right|^{g} \mu_{L}\right\|_{1}=\left\|\mu _ { L } \left|\left\|_{1},||^{g} \mu_{R}\right\|_{1}=\left\|\mu_{R}\right\|_{1},\left\|{ }^{g} \mu\right\|_{1}=\|\mu\|_{1} .\right.\right.
\end{array}\right.
$$

Then $\mathfrak{X}_{L}^{*, A}, \mathfrak{X}_{R}^{*, A}, \mathfrak{X}^{*, \perp^{\prime \prime}}$ become Banach algebras with unit (the unit being $\delta_{e}$, the Dirac measure at the neutral element $e$ of $G$ ) if we define the $\sigma$-convolution of measures according to [2], [8]:

$$
\begin{aligned}
& \left\{\begin{array}{l}
\left\langle\mu_{R} *^{\sigma} \nu_{L}, h\right\rangle=\left\langle\mu_{L}(u),\left\langle{ }^{u} \nu_{L}(v), h(u v)\right\rangle, \mu_{L}, \nu_{L} \in \mathfrak{X}_{L}^{*, A}\right. \\
\left\langle\mu *^{\sigma} \nu, h\right\rangle=\left\langle\mu(u),\left\langle{ }^{u} \nu(v), h(u v)\right\rangle, \mu, \nu \in \mathfrak{X}^{*, d}{ }^{\prime \prime}\right.
\end{array}\right. \\
& \left\{\begin{array}{l}
\left.\left\langle h, \mu_{R} *^{\sigma} \nu_{R}\right\rangle=\left\langle h(u v),{ }^{u} \nu_{R}(v)\right\rangle, \mu_{R}(u)\right\rangle, \mu_{R}, \nu_{R} \in \mathfrak{X}_{R}^{*, A} \\
\left\langle h, \mu *^{\sigma} \nu\right\rangle=\left\langle\left\langle h(u v),{ }^{u} \nu(v)\right\rangle, \mu(u)\right\rangle .
\end{array}\right.
\end{aligned}
$$

Through the correspondence

$$
f \in L^{1}(G, A) \longrightarrow \mu_{f}:\left\langle\mu_{f}, \varphi\right\rangle=\int f(g) \varphi(g) d g, \varphi \in K(G)
$$

the Banach space $L^{1}(G, A)$ (for the norm $\left.\|f\|_{1}=\int|f(g)| d g\right)$ of functions from $G$ to $A$, Bochner-integrable with respect to the Haar measure, can be identified with a left ideal (resp. right ideal, subalgebra) of $\mathfrak{X}_{L}^{*, A}$ (resp. $\mathfrak{X}_{R}^{*, A}, \mathfrak{X}^{*, A^{\prime \prime}}$ ) and we have the following formulas, where $\delta$ is the modular function of $G$ :

$$
\begin{aligned}
f_{1} *^{\top} f_{2} & =\int f_{1}(u) \sigma(u) f_{2}\left(u^{-1} \cdot\right) d u \\
& =\int f_{1}(\cdot u) \sigma(\cdot u) f_{2}\left(u^{-1}\right) d u \\
& =\int \delta\left(u^{-1}\right) f_{1}\left(\cdot u^{-1}\right) \sigma\left(\cdot u^{-2}\right) f_{2}(u) d u \\
& =\int \delta\left(u^{-1}\right) f_{2}\left(u^{-1}\right) \sigma\left(u^{-1}\right) f_{2}\left(u^{-1} \cdot\right) d u
\end{aligned}
$$


In the sequel, we will usually omit the subscript $\sigma$ to denote the $\sigma$-convolution. $L^{1}(G, A)$ may be called the Banach crossed product of $G$ by $A$ [4], [8] and the following theorem can be proved:

\section{THEOREM 2. ([2], Theorems 4.10, 4.15, 4.19.)}

Let $A$ be a Banach-*-algebra, $A^{\prime \prime}$ its enveloping Von Neumann algebra when $A$ is a $C^{*}$-algebra.

$$
\left\{\begin{array}{l}
M_{L}\left(L^{1}(G, A)\right)=\mathfrak{X}_{R}^{*, A} \\
M_{R}\left(L^{1}(G, A)\right)=\mathfrak{X}_{R}^{*, A} \\
M\left(L^{1}(G, A)\right)=\mathfrak{X}^{*, A}
\end{array}\right.
$$

where $\mathfrak{X}^{*, A}$ (in general different from $M_{1}(G, M(A)$ ) is the idealizer of $L^{1}(G, A)$ in $\mathfrak{X}_{L}^{* A}$ or $\mathfrak{X}_{R}^{*, A}$. $L^{1}(G, A), \mathfrak{X}^{*, A}$ and $\mathfrak{X}^{*, A^{\prime \prime}}$ are Banach-*-algebras if we define

$$
\left\{\begin{array}{l}
f^{*}(g)=\delta\left(g^{-1}\right) \sigma(g) f\left(g^{-1}\right)^{*} \\
\left(\mu_{L} * f\right)^{*}=f^{*} * \mu_{L}^{*} ;\left(f * \mu_{R}\right)^{*}=\mu_{R}^{*} f^{*},(\mu * f)^{*}=f^{*} * \mu^{*}
\end{array}\right.
$$

and $L^{1}(G, A)$ is a *-ideal (resp. *-subalgebra) of $\mathfrak{X}^{*, A}$ (resp. $\mathfrak{X}^{*, A^{\prime \prime}}$ ).

3. Module structures on $\mathfrak{X}, \mathfrak{X}_{L}^{*, A}, \mathfrak{X}_{R}^{*, A}, \mathfrak{X}^{*, A}, \mathfrak{X}^{*, A^{\prime \prime}}$. In this paragraph, we are going to define several natural module structures on the various objects we introduced in the preceding one. We first begin with $G$-module structures.

Proposition 1. $\mathfrak{X}$ is a Banach-G-module in two different ways corresponding to the two following different actions of $G$, denoted successively by $a$. and by $a \circ$ :

$$
\begin{gathered}
g \cdot h=h * \delta_{g^{-1}}=h(\cdot g) ; h \cdot g=\sigma(g)\left\{\delta_{g^{-1}}\right\} * h=h(g \cdot), h \in \mathfrak{X}, g \in G \\
g \circ h=g \cdot h ; h \circ g=h .
\end{gathered}
$$

Proof. First of all $g^{\prime} \cdot(g \cdot h)=\left(g^{\prime} g\right) \cdot h$ and $\|g \cdot h\|_{\infty}=\|h\|_{\infty}$. Then, given $\varepsilon$, let $k \in K(G, A)$ such that $\|h-k\|_{\infty} \leqq \varepsilon / 3$ and $V(e)$ a neighborhood of $e$ in $G$ such that $\|g \cdot k-k\|<\varepsilon / 3$ when $g \in V(e)$. Then $\left\|g \cdot h-\left.h\right|_{\infty} \leqq\right\| g \cdot h-g \cdot k\left\|_{\infty}+\right\| g \cdot k-k\left\|_{\infty}+\right\| k-h \|_{\infty} \leqq \varepsilon$ when $g \epsilon$ $V(e)$. Same proof for the right action.

Proposition 2. $\mathfrak{X}_{L}^{*, A}, \mathfrak{X}_{R}^{*, A}, \mathfrak{X}^{*, A}$ and $\mathfrak{X}^{*, A^{\prime \prime}}$ are G-modules in two different ways corresponding to the two following different actions of $G$, denoted successively by $a$. and by $\circ$ : 
(21)

$$
\left\{\begin{array}{l}
g \cdot \mu_{L}=\delta_{g} * \mu_{L} ; \mu_{L} \cdot g=\mu_{L} * \delta_{g}, \mu_{L} \in \mathfrak{X}_{L}^{*, A}, g \in G \\
g \cdot \mu_{R}=\delta_{g}^{*} \mu_{R} ; \mu_{R} \cdot g=\mu_{R} * \delta_{g} ; \mu_{R} \in \mathfrak{X}_{R}^{*, A}, g \in G \\
g \cdot\left(\mu_{L}, \mu_{R}\right)=\left(g \cdot \mu_{L}, g \cdot \mu_{R}\right) ; \\
\left(\mu_{L}, \mu_{R}\right) \cdot g=\left(\mu_{L} \cdot g, \mu_{R} \cdot g\right),\left(\mu_{L}, \mu_{R}\right) \in \mathfrak{X}^{*, A}, g \in G \\
g \cdot \mu=\delta_{g} * \mu ; \mu \cdot g=\mu * \delta_{g}, \mu \in \mathfrak{X}^{*, A}, g \in G .
\end{array}\right.
$$

$\left(21^{\text {bis }}\right)$

$$
\left\{\begin{array}{l}
g \circ \mu_{L}=\mu_{L} ; \mu_{L} \circ g=\mu_{L} \cdot g \\
g \circ \mu_{R}=\mu_{R} ; \mu_{R} \circ g=\mu_{R} \cdot g \\
g \circ\left(\mu_{L}, \mu_{R}\right)=\left(\mu_{L}, \mu_{R}\right) ;\left(\mu_{L}, \mu_{R}\right) \circ g=\left(\mu_{L} \circ g, \mu_{R} \circ g\right) \\
g \circ \mu=\mu ; \mu \circ g=\mu \circ g .
\end{array}\right.
$$

Proof. The proof is straightforward, and left to the reader.

With the same notations, we then have the following formulas, relating these $G$-module structures:

\section{Proposition 3.}

$$
\begin{aligned}
& (22) \quad\left\{\begin{array}{l}
\left\langle g \cdot \mu_{L}, h\right\rangle=\left\langle{ }^{g} \mu_{L}, h \cdot g\right\rangle ;\left\langle h, g \cdot \mu_{R}\right\rangle=\left\langle h \cdot g,{ }^{g} \mu_{R}\right\rangle \\
\langle g \cdot \mu, h\rangle=\left\langle{ }^{g} \mu, h \cdot g\right\rangle ;\langle h, g \cdot \mu\rangle=\langle h \cdot g, \mu\rangle
\end{array}\right. \\
& (23) \quad\left\{\begin{array}{l}
\left\langle\mu_{L} \cdot g, h\right\rangle=\left\langle\mu_{L}, g \cdot h\right\rangle ;\left\langle h, \mu_{R} \cdot g\right\rangle=\left\langle g \cdot h, \mu_{R}\right\rangle \\
\langle\mu \cdot g, h\rangle=\langle\mu, g \cdot h\rangle ;\langle h, \mu \cdot g\rangle=\langle g \cdot h, \mu\rangle
\end{array}\right. \\
& \left(22^{\text {bis }}\right) \quad\left\{\begin{array}{l}
\left\langle g \circ \mu_{L}, h\right\rangle=\left\langle\mu_{L}, h \circ g\right\rangle=\left\langle\mu_{L}, h\right\rangle ; \\
\left\langle h, g \circ \mu_{R}\right\rangle=\left\langle h \circ g, \mu_{R}\right\rangle=\left\langle h, \mu_{R}\right\rangle \\
\langle g \circ \mu, h\rangle=\langle\mu, h \circ g\rangle=\langle\mu, h\rangle ;\langle h, g \circ \mu\rangle=\langle h \circ g, \mu\rangle=\langle h, \mu\rangle
\end{array}\right. \\
& \left(23^{\text {bis }}\right) \quad\left\{\begin{array}{l}
\left\langle\mu_{L} \circ g, h\right\rangle=\left\langle\mu_{L}, g \circ h\right\rangle ;\left\langle h, \mu_{R^{\circ}} \circ\right\rangle=\left\langle g \circ h, \mu_{R}\right\rangle \\
\langle\mu \circ g, h\rangle=\langle\mu, g \circ h\rangle ;\left\langle h, \mu \mu^{\circ} g\right\rangle=\langle g \circ h, \mu\rangle
\end{array}\right. \\
& (24) \quad\left\|\mu_{L} \cdot g\right\|_{1}=\left\|g \cdot \mu_{L}\right\|_{1}=\left\|\mu_{L}\right\|_{1} ;\left\|\mu_{R} \cdot g\right\|=\left\|g \cdot \mu_{R}\right\|_{1}=\left\|\mu_{R}\right\|_{1} ; \\
& \|g \cdot \mu\|_{1}=\|\mu \cdot g\|_{1}=\|\mu\|_{1} .
\end{aligned}
$$

Proof. Formulas (22), (23), $\left(22^{\text {bis }}\right),\left(23^{\text {bis }}\right)$ are just a matter of computation. Let us prove (24): with notations of (6),

$$
\begin{aligned}
& \left\|\mu_{L} \cdot g\right\|_{1}=\|\| U_{\mu_{L}} \cdot g \|=\sup \sum_{i}\left|U_{\mu_{L} \cdot g}\left(h_{i}\right)\right|=\sup \sum_{i}\left|U_{\mu_{L}}\left(g \cdot h_{i}\right)\right| \\
& =\sup \sum_{i}\left|U_{n_{L}}\left(h_{i}\right)\right|=\left|\left\|U_{n_{L}} \mid\right\|=\left\|\mu_{L}\right\|_{1}\right. \\
& \left\|g \cdot \mu_{L}\right\|_{1}=\|\| U_{g \cdot \mu_{L}}\left|\|=\sup \sum_{i}\right| U_{g \cdot \mu_{L}}\left(h_{i}\right)\left|=\sup \sum_{i}\right| U_{g_{\mu_{L}}}\left(h_{i} \cdot g\right) \mid \\
& =\sup \sum_{i}\left|U_{g^{\prime} L}\left(h_{i}\right)\right|=\|\| U_{g^{\prime} L} \mid\|=\|{ }^{g} \mu_{L}\left\|_{1}=\right\| \mu_{L} \|_{1}
\end{aligned}
$$

and the same for $\mu_{R}$ and $\mu$. 
Proposition 4. The functions $g \rightarrow g \cdot \mu_{L}, g \rightarrow \mu_{L} \cdot g$ (resp. $g \rightarrow g \cdot \mu_{R}$, $\left.g \rightarrow \mu_{R} \cdot g ; g \rightarrow g \cdot \mu, g \rightarrow \mu \cdot g\right)$ are continuous ,in the $\mathfrak{X}$-weak-topology, i.e., the topology defined by the semi-norms: $\left|\mu_{L}\right|_{h}=\left|\left\langle\mu_{L}, h\right\rangle\right|$ (resp. $\left|\mu_{R}\right|^{h}=\left|\left\langle h, \mu_{R}\right\rangle\right| ;|\mu|_{h}$ and $\left.|\mu|^{h}\right)$.

Proof. We give the proof for the first function only. Then, given $\mu_{L}, h$ and $\varepsilon>0$, there exists $V(e)$, neighborhood of $e$ in $G$, such that, if $g \in V(e),\|h \cdot g-h\|_{\infty}<\varepsilon / 3\left\|\mu_{L}\right\|_{1},\left|\left\langle\mu_{L}, h\right\rangle=\sigma\left(g^{-1}\right)\left\langle\mu_{L}, h\right\rangle\right|<$ $\varepsilon / 3$ by continuity of $\sigma,\left|\left\langle\mu_{L}, \sigma\left(g^{-1}\right) h-h\right\rangle\right|<\varepsilon / 3$ by second countability of $G$ and Lebesgue's dominated convergence theorem. Hence,

$$
\begin{aligned}
&\left|\left\langle g \cdot \mu_{L}-\mu_{L}, h\right\rangle\right|=\left|\left\langle g \mu_{L}, h \cdot g\right\rangle-\left\langle\mu_{L}, h\right\rangle\right|=\mid \sigma(g)\left\langle\mu_{L}, \sigma\left(g^{-1}\right) h(g \cdot)\right\rangle \\
& \quad-\left\langle\mu_{L}, h\right\rangle|=|\left\langle\mu_{L}, \sigma\left(g^{-1}\right) h(g \cdot)\right\rangle-\sigma\left(g^{-1}\right)\left\langle\mu_{L}, h\right\rangle \mid \\
&=\left|\left\langle\mu_{L}, \sigma\left(g^{-1}\right) h(g \cdot)-\sigma\left(g^{-1}\right) h+\sigma\left(g^{-1}\right) h-h+h\right\rangle-\sigma\left(g^{-1}\right)\left\langle\mu_{L}, h\right\rangle\right| \\
& \leqq\left\langle\mu_{L}, \sigma\left(g^{-1}\right) h(g \cdot)-\sigma\left(g^{-1}\right) h\right\rangle|+|\left\langle\mu_{L}, \sigma\left(g^{-1}\right) h-h\right\rangle \mid \\
&+\left|\left\langle\mu_{L}, h\right\rangle-\sigma\left(g^{-1}\right)\left\langle\mu_{L}, h\right\rangle\right| \leqq\left\|\mu_{L}\right\|_{1}\|h \cdot g-h\|_{\infty}+\left|\left\langle\mu_{L}, \sigma\left(g^{-1}\right) h-h\right\rangle\right| \\
&+\left|\left\langle\mu_{L}, h\right\rangle-\sigma\left(g^{-1}\right)\left\langle\mu_{L}, h\right\rangle\right|<\varepsilon .
\end{aligned}
$$

In a second step, we now introduce more general module structures.

Proposition 5. $\mathfrak{X}$ becomes a unital Banach- $\mathfrak{X}_{L}^{*, A}$ (or $\mathfrak{X}_{R}^{*, A}$, or $\mathfrak{X}^{*, A}$ )module (and a neo-unital Banach- $L^{1}(G, A)$-module by restriction) according to

$$
\left\{\begin{array}{l}
\mu_{L} \circ h=\mu_{L} \cdot h, h \circ \mu_{L}=\mu_{L} \\
\mu_{R} \circ h=\mu_{R} \cdot h, h \circ \mu_{R}=\mu_{R}
\end{array}\right.
$$

where

$$
\left\{\begin{aligned}
\left(\mu_{L} \cdot h\right)(g) & =\left\langle{ }^{g} \mu_{L}(u), u \cdot h(g)\right\rangle=\left\langle{ }^{g} \mu_{L}, h \cdot g\right\rangle \\
& =\left\langle g \cdot \mu_{L}, h\right\rangle=\left\langle\delta_{g} * \mu_{L}, h\right\rangle \\
\left(\mu_{R} \cdot h\right)(g) & =\left\langle u \cdot h(g),{ }^{g} \mu_{R}(u)\right\rangle=\left\langle h \cdot g,{ }^{g} \mu_{R}\right\rangle \\
& =\left\langle h, g \cdot \mu_{R}\right\rangle=\left\langle h, \delta_{g} * \mu_{R}\right\rangle .
\end{aligned}\right.
$$

Then

$$
\begin{aligned}
& \left\|\mu_{L} \cdot h\right\|_{\infty} \leqq\left\|\mu_{L}\right\|_{1}\|h\|_{\infty} ;\left\|\mu_{R} \cdot h\right\|_{\infty} \leqq\left\|\mu_{R}\right\|_{1}\|h\|_{\infty} \\
& \mu_{L} \cdot\left(\nu_{L} \cdot h\right)=\left(\mu_{L} * \nu_{L}\right) \cdot h ; \mu_{R} \cdot\left(\nu_{R} \cdot h\right)=\left(\mu_{R} * \nu_{R}\right) \cdot h
\end{aligned}
$$

and, in particular

$$
\delta_{g} \cdot h=g \cdot h, \delta_{0} \cdot h=h .
$$

If $\left\{\lambda_{\beta}\right\}_{\beta \in J}$ is a countable approximate unit in $L^{1}(G, A)$, then 


$$
\mu_{L} \cdot h=\lim _{\alpha, \beta}\left(\mu_{L} * e_{\alpha} \lambda_{\beta}\right) \cdot h ; \mu_{R} \cdot h=\lim _{\alpha, \beta}\left(\mu_{R} * e_{\alpha} \lambda_{\beta}\right) \cdot h .
$$

Proof. $\left(\mu_{L} \cdot h\right)(g)$ is continuous by Proposition 4. Moreover, as $\mu_{L}$ is a regular Borel measure, given $\varepsilon>0$, there exists a compact $K \subset G$ such that $\left|\mu_{L}\right|(G / K)<\varepsilon / 2\|h\|_{\infty}$ for $h \in \mathfrak{X}$. Given $\varepsilon>0$ and $K$, there exists a compact $K^{\prime}$ such that, if $g \notin K^{\prime}, \sup _{u \in K}|h(g u)|<$ $\varepsilon / 2\left\|\mu_{L}\right\|_{1}$. So

$$
\begin{aligned}
\left|\left(\mu_{L} \cdot h\right)(g)\right| & \leqq\left\langle\left|\mu_{L}\right|,|h(g \cdot)|\right\rangle \\
& =\int_{K} d\left|\mu_{L}\right|(u)|h(g u)|+\int_{G / K} d\left|\mu_{L}\right|(u)|h(g u)| \\
& \leqq\left\|\mu_{L}\right\|_{1} \sup _{u \in K}|h(g u)|+\left|\mu_{L}\right|(G / K) \cdot\|h\|_{\infty} \leqq \varepsilon \text { if } g \notin K^{\prime},
\end{aligned}
$$

and then $\mu_{L} \cdot h \in \mathfrak{X}$, and also $\mu_{R} \cdot h \in \mathfrak{X}$. Formulas (26) and (29) are just a matter of computation. Moreover (29) proves the unital character of $\mathfrak{X}$, while its neo-unital character on $L^{1}(G, A)$ comes from (26): more precisely (26) shows the set $\left\{e_{\alpha} \lambda_{\beta} \cdot h\right\}_{\alpha \in I, \beta \in J}$ is dense in $\mathfrak{X}$, while the Curtis-Figa-Talamanca factorization theorem proves this set generates a closed subspace of $\mathfrak{X}([6]$, p. 169-185). Hence any $h \in \mathfrak{X}$ can be written $h=f \cdot h^{\prime}$ with $h^{\prime} \in \mathfrak{X}$ and $f \in L^{1}(G, A)$. This allows to prove (30) because

$$
\begin{aligned}
\lim _{\alpha, \beta}\left(\mu_{L} * e_{\alpha} \lambda_{\beta}\right) \cdot h & =\lim _{\alpha, \beta}\left(\mu_{L} * e_{\alpha} \lambda_{\beta}\right) \cdot f \cdot h^{\prime}=\lim _{\alpha, \beta}\left(\mu_{L} * e_{\alpha} \lambda_{\beta} * f\right) \cdot h^{\prime} \\
& =\left(\mu_{L} * f\right) \cdot h^{\prime}=\mu_{L} \cdot f \cdot h^{\prime}=\mu_{L} \cdot h .
\end{aligned}
$$

It does not seem possible to define a nontrivial action of $\mathfrak{X}_{L}^{*, A}$ or $\mathfrak{X}_{R}^{*}$ on the right of $\mathfrak{X}$ which turns it into a Banach module. Formula (30) of the preceding theorem means the action of $x_{R}^{*}, A$ or $\mathfrak{X}_{R}^{*, A}$ can be deduced from the one of $L_{1}(G, A)$ by extension to its left and right centralizers.

Proposition 6. $\mathfrak{X}_{L}^{*, A}, \mathfrak{X}_{R}^{*}, \mathfrak{X}^{*, 4}$ and $\mathfrak{X}^{*, 1^{\prime \prime}}$ are unital Banach modules onto themselves in two different ways, corresponding to the two following different actions, denoted successively by $a \cdot$ and by $a \circ$ :

$$
\begin{aligned}
& \left\{\begin{array}{l}
\left(\nu_{L} \cdot \mu_{L}=\nu_{L} * \mu_{L} ; \mu_{L} \cdot \nu_{L}=\mu_{L} * \nu_{L}\right. \\
\nu_{R} \cdot \mu_{R}=\nu_{R} * \mu_{R} ; \mu_{R} \cdot \nu_{R}=\mu_{R} * \nu_{R} \\
\left(\nu_{L}, \nu_{R}\right) \cdot\left(\mu_{L}, \mu_{R}\right)=\left(\nu_{L} * \mu_{L}, \nu_{R} * \mu_{R}\right) ;\left(\mu_{L}, \mu_{R}\right) \cdot\left(\nu_{L}, \nu_{R}\right)=\left(\mu_{L} * \nu_{L}, \mu_{R} * \nu_{R}\right) \\
\nu \cdot \mu=\nu * \mu ; \mu \cdot \nu=\mu * \nu
\end{array}\right. \\
& \left\{\begin{array}{l}
\nu_{L} \circ \mu_{L}=\mu_{L} ; \mu_{L} \circ \nu_{L}=\mu_{L} * \mu_{L} \\
\nu_{R} \circ \mu_{R}=\mu_{R} ; \mu_{R} \circ \nu_{R}=\mu_{R} * \nu_{R}
\end{array}\right.
\end{aligned}
$$




$$
\left\{\begin{array}{l}
\left(\nu_{L}, \nu_{R}\right) \circ\left(\mu_{L}, \mu_{R}\right)=\left(\mu_{L}, \mu_{R}\right) ;\left(\mu_{L}, \mu_{R}\right) \circ\left(\nu_{L}, \nu_{R}\right)=\left(\mu_{L} * \nu_{L}, \mu_{R} * \nu_{R}\right) \\
\nu \circ \mu=\mu ; \mu \circ \nu=\mu * \nu .
\end{array}\right.
$$

Proof. The proof is straightforward and left to the reader.

With the same notations, we then have the following formulas, relating these module structures:

Proposition 7.

$$
\left\langle\mu_{L} \cdot \nu_{L}, h\right\rangle=\left\langle\mu_{L}, \nu_{L} \cdot h\right) ;\left\langle h, \mu_{R} \cdot \nu_{R}\right\rangle=\left\langle\nu_{R} \cdot h, \mu_{R}\right\rangle
$$

$\left(32^{1 \mathrm{is}}\right) \quad\left\{\begin{array}{l}\left\langle\nu_{L} \circ \mu_{L}, h\right\rangle=\left\langle\mu_{L}, h \circ \nu_{L}\right\rangle=\left\langle\mu_{L}, h\right\rangle ;\left\langle h, \nu_{R} \circ \mu_{R}\right\rangle=\left\langle h \circ \nu_{R}, \mu_{R}\right\rangle=\left\langle h, \mu_{R}\right\rangle \\ \left\langle\mu_{L} \circ \nu_{L}, h\right\rangle=\left\langle\mu_{L}, \nu_{L} \circ h\right\rangle ;\left\langle h, \mu_{R} \circ \nu_{R}\right\rangle=\left\langle\nu_{R} \circ h, \mu_{R}\right\rangle .\end{array}\right.$

According to formulas (22), (23) and (32) one side, and $\left(22^{\text {bis }}\right)$, $\left(23^{\text {bis }}\right)$ and $\left(32^{\text {bir }}\right)$ on the other side, we can refer to the structure denoted by $a$ - as the "nondual" structure, and the structure denoted by a o as the "dual" structure.

4. Homology and cohomology over $L_{1}(G, A)$. In the sequel, we will denote, for convenience,

$$
\mathfrak{A}=L^{1}(G, A)
$$

and

$$
L_{n}(\mathfrak{H}, \mathfrak{X})=\mathfrak{A} \hat{\otimes} \mathfrak{A} \hat{\otimes} \cdots \hat{\otimes} \mathfrak{A} \hat{\otimes} \mathfrak{X}=\mathfrak{H}^{\hat{\otimes} n} \hat{\otimes} \mathfrak{X}
$$

where there are $n$ copies of $\mathfrak{A}(n \geqq 0)$ and where $\hat{\otimes}$ denotes the projective tensor product [11].

Let us define the application $D_{n}$ from $L_{n}(\mathfrak{A}, \mathfrak{X})$ into $L_{n-1}(\mathfrak{A}, \mathfrak{X})$ by the continuous linear extension of

$$
\begin{gathered}
D_{n}\left(f_{1} \otimes f_{1} \otimes f_{2} \otimes \cdots \otimes f_{n} \otimes h\right)=f_{2} \otimes f_{3} \otimes \cdots \otimes f_{n} \otimes h \\
\quad+\sum_{i=1}^{n-1}(-1)^{i} f_{1} \otimes \cdots \otimes f_{i}^{*} f_{i+1} \otimes \cdots f_{n} \otimes h \\
\quad+(-1)^{n} f_{1} \otimes f_{2} \otimes \cdots \otimes f_{n-1} \otimes\left(f_{n} \circ h\right)
\end{gathered}
$$

where $f_{i} \in \mathfrak{A}, \quad i=1, \cdots, n$ and $h \in \mathfrak{X} . \quad\left(D_{0}\right.$ is defined as the null application.) Then $D_{n} D_{n+1}=0$ for all $n \geqq 0$ and it is possible to introduce the quotient space

$$
H_{n}(\mathfrak{A}, \mathfrak{X})=\frac{\operatorname{Ker} D_{n}}{\operatorname{Im} D_{n+1}} .
$$

One can notice that it is possible to write 


$$
L_{n}(\mathfrak{A}, \mathfrak{X})=L^{1}(G, A)^{\hat{\otimes} n} \hat{\otimes} \mathfrak{X}=L^{1}\left(G^{n}\right) \otimes A^{\hat{\otimes}^{n}} \otimes \mathfrak{X}
$$

or

$$
L_{n}(\mathfrak{A}, \mathfrak{X})=L^{1}\left(G^{n}, A^{\hat{\otimes} n}\right) \hat{\otimes} \mathfrak{X}
$$

or

$$
L_{n}(\mathfrak{A}, \mathfrak{X})=L^{1}\left(G^{n}, A^{\hat{\otimes} n} \otimes \mathfrak{X}\right) .
$$

Let now $L_{n}(\mathfrak{A}, \mathfrak{X})_{L}^{*, A}$ be the space of functionals $T$ on $L_{n}(\mathfrak{A}, \mathfrak{X})$ with values in $A$ which are $n$-linear on $\mathfrak{A}, A$-right linear on $\mathfrak{X}$, and bounded in the sense that, for any finite family $\left(h_{i}\right)$ of functions $h_{i} \in K(G, A)$ with support $h_{i} \cap$ support $h_{j}=\varnothing$ for any $i \neq j$,

$$
\sum_{i}\left|T\left(f_{1} \otimes \cdots \otimes f_{n} \otimes h_{i}\right)\right| \leqq K\left\|f_{1}\right\|_{1} \cdots\left\|f_{n}\right\|_{1}\left\|\Sigma h_{i}\right\|_{\infty}
$$

where $K$ is some constant.

Hence it is possible to identify $L_{n}(\mathfrak{A}, \mathfrak{X})_{L}^{*, A}$ with $L^{n}\left(\mathfrak{A} \cdot \mathfrak{X}_{L}^{*, A}\right)$, the space of continuous $n$-linear functionals on $\mathfrak{A}$ with values in $\mathfrak{X}_{L}^{*, A}$ according to (we use the same letter $T$ to denote the two corresponding objects):

$$
T\left(f_{1} \otimes \cdots \otimes f_{n} \otimes h\right)=\left\langle T\left(f_{1} \otimes \cdots \otimes f_{n}\right), h\right\rangle .
$$

In the same way, we could introduce $L^{n}\left(\mathfrak{A}, \mathfrak{X}_{R}^{*, A}\right), L^{n}\left(\mathfrak{A}, \mathfrak{X}^{*, A}\right)$ and $L^{n}\left(\mathfrak{A}, \mathfrak{X}^{*, 4^{\prime \prime}}\right)$.

Let us now define the application $\Delta^{n}$ from

$$
L^{n-1}\left(\mathfrak{A}, \mathfrak{X}_{L}^{* A}\right)\left(\operatorname{resp} . L^{n-1}\left(\mathfrak{A}, \mathfrak{X}_{R}^{*, A}\right), L^{n-1}\left(\mathfrak{A}, \mathfrak{X}^{*, A}\right), L^{n-1}\left(\mathfrak{A}, \mathfrak{X}^{*, A^{\prime \prime}}\right)\right)
$$

into

$$
L^{n}\left(\mathfrak{A}, \mathfrak{X}_{L}^{*, A}\right)\left(\operatorname{resp} . L^{n}\left(\mathfrak{A}, \mathfrak{X}_{R}^{*, A}\right), L^{n}\left(\mathfrak{A}, \mathfrak{X}^{*, A}\right), L^{n}\left(\mathfrak{A}, \mathfrak{X}^{*, A^{\prime \prime}}\right)\right)
$$

by the following formula, corresponding to the "nondual" structure:

$$
\begin{aligned}
\Delta^{n} T\left(f_{1} \otimes\right. & \left.\cdots \otimes f_{n}\right)=f_{1} \cdot T\left(f_{2} \otimes \cdots \otimes f_{n}\right) \\
& +\sum_{i=1}^{n-1}(-1)^{i} T\left(f_{1} \otimes \cdots \otimes f_{i} * f_{i+1} \otimes \cdots \otimes f_{n}\right) \\
& +(-)^{n} T\left(f_{1} \otimes \cdots \otimes f_{n-1}\right) \cdot f_{n} .
\end{aligned}
$$

Then $\Delta^{n+1} \Delta^{n}=0$ and it is possible to introduce the quotient space

$$
H^{n}\left(\mathfrak{A}, \mathfrak{X}_{L}^{*, A}\right)=\frac{\operatorname{Ker} \Delta^{n+1}}{\operatorname{Im} \Delta^{n}}=\frac{Z^{n}\left(\mathfrak{A}, \mathfrak{X}_{L}^{*, A}\right)}{N^{n}\left(\mathfrak{A}, \mathfrak{X}_{L}^{*, A}\right)}
$$

and, in the same way, $H^{n}\left(\mathfrak{A}, \mathfrak{X}_{R}^{*, A}\right), H^{n}\left(\mathfrak{A}, \mathfrak{X}^{*, A}\right), H^{n}\left(\mathfrak{A}, \mathfrak{X}^{*, A^{\prime \prime}}\right)$.

In the "dual" structure case, we have to modify slightly our 
definitions, asking for $T$ to be $n$-affine and continuous on $\mathfrak{A}$, and replacing formula (42) by

$$
\begin{aligned}
\Delta^{n} T\left(f_{1} \otimes\right. & \left.\cdots \otimes f_{n}\right)=T\left(f_{2} \otimes \cdots \otimes f_{n}\right) \\
& +\sum_{i=1}^{n-1}(-1)^{i} T\left(f_{1} \otimes \cdots \otimes f_{i} * f_{i+1} \otimes \cdots \otimes f_{n}\right) \\
& +(-1)^{n} T\left(f_{1} \otimes \cdots \otimes f_{n-1}\right) \circ f_{n} .
\end{aligned}
$$

In that case, it is easy to prove the "duality" formula:

$$
\left\langle T, D_{n}\left(f_{1} \otimes \cdots \otimes f_{n} \otimes h\right)\right\rangle=\left\langle\Delta^{n} T, f_{1} \otimes \cdots \otimes f_{n} \otimes h\right\rangle
$$

and we have the following theorem, relating (36) and (43) in the "dual" case.

THEOREM 3. Let us assume there exists an $F \in A^{\prime}$ such that $F(a)=0$ imply $a=0$ (it is the case if $A$ is separable). Then $H_{n}(\mathfrak{A}, \mathfrak{X})=0$ and $\operatorname{Im} D_{n}$ closed is, in the "dual" case, equivalent to $H^{n}\left(\mathfrak{A}, \mathfrak{X}_{L}^{* A}\right)=0, H^{n}\left(\mathfrak{A}, \mathfrak{X}_{L^{*}}^{*}\right)=0, H^{n}\left(\mathfrak{A}, \mathfrak{X}^{*, A}\right)=0$ or $H^{n}\left(\mathfrak{A}, \mathfrak{X}^{*, A^{\prime \prime}}\right)=0$.

Proof. Let $L^{n}\left(\mathfrak{A}, \mathfrak{X}^{\prime}\right)$ the space of continuous $n$-linear functionals on $\mathfrak{A}$ with value in $\mathfrak{X}^{\prime}$ (the dual of $\mathfrak{X}$ ), i.e., the dual of $L_{n}(\mathfrak{A}, \mathfrak{X})$. Given $T \in L^{n}\left(\mathfrak{H}, \mathfrak{X}_{L^{*}}^{*}\right)$ and $F \in A^{\prime}$ let $T_{F}$ be the element of $L^{n}\left(\mathfrak{A}, \mathfrak{X}^{\prime}\right)$ defined by

$$
\left\langle T_{F}, f_{1} \otimes \cdots \otimes f_{n} \otimes h\right\rangle=F\left\{\left\langle T, f_{1} \otimes \cdots \otimes f_{n} \otimes h\right\rangle\right\}
$$

and let $L^{n}\left(\mathfrak{A}, \mathfrak{X}_{L}^{*}\right)_{F}$ be the closed subspace of $L^{n}\left(\mathfrak{A}, \mathfrak{X}^{\prime}\right)$ generated by the set of $T_{F}$ with $T \in L^{n}\left(\mathfrak{A}, \mathfrak{X}_{L}^{*}{ }^{A}\right)$. By faithfulness of $F$, the correspondence $T \rightarrow T_{F}$ is injective and the spaces $L_{n}(\mathfrak{A}, \mathfrak{X})$ and $L^{n}\left(\mathfrak{A}, \mathfrak{X}_{L}^{*, A}\right)_{F}$ are in duality. Moreover, if $\Delta_{1}^{n}$ means the equivalent of $\Delta^{n}$ on $L^{n}\left(\mathfrak{A}, \mathfrak{X}^{\prime}\right)$, we have, if we define $f \circ T_{F}=T_{F}$ and $T_{F^{\circ}} \circ f=$ $(T \circ f)_{F}$,

$$
\begin{aligned}
\left\langle T_{F},\right. & \left.D^{n+1}\left(f_{1} \otimes \cdots \otimes f_{n+1} \otimes h\right)\right\rangle=\left\langle\Delta_{1}^{n+1} T_{F}, f_{1} \otimes \cdots \otimes f_{n+1} \otimes h\right\rangle \\
& =F\left\{\left\langle T, D^{n+1}\left(f_{1} \otimes \cdots \otimes f_{n+1} \otimes h\right)\right\rangle\right\}=F\left\{\left\langle\Delta^{n+1} T, f_{1} \otimes \cdots \otimes f_{n} \otimes h\right\rangle\right\} \\
& =\left\langle\left(\Delta^{n+1} T\right)_{F}, f_{1} \otimes \cdots \otimes f_{n+1} \otimes h\right\rangle .
\end{aligned}
$$

So $\Delta_{1}^{n+1} T_{F}=\left(\Delta^{n+1} T\right)_{F}: \Delta_{1}^{n+1}$, when restricted to $L^{n}\left(\mathfrak{A}, \mathfrak{X}_{L}^{*}{ }^{A}\right)_{F}$, maps it into $L^{n+1}\left(\mathfrak{A}, \mathfrak{X}_{L}^{*}, A\right)$, and is the transpose of $D^{n+1}$ in the duality $\left\langle L_{n}(\mathfrak{A}, \mathfrak{X}), L^{n}\left(\mathfrak{A}, \mathfrak{X}_{L}^{*, A}\right)_{F}\right\rangle$. Hence, in the same way as in ([15], Corollary 1.3), it is possible to prove the theorem is true if $H^{n}\left(\mathfrak{A}, \mathfrak{X}_{i,}^{* .4}\right)$ is replaced by $H^{n}\left(\mathfrak{A}, \mathfrak{X}_{L}^{*}\right)_{F}$.

But let us now assume that $H^{n}\left(\mathfrak{A}, \mathfrak{X}_{L}^{*}{ }^{4}\right)=0$, that is to say that $\Delta^{n+1} T=0$ imply $T=\Delta^{n} T^{\prime}$ with $T^{\prime} \in L^{n-1}\left(\mathfrak{A}, \mathfrak{X}_{L}^{*}{ }^{A}\right)$. By faithfulness of $F, \Delta^{n+1} T=0$ is equivalent to $\Delta_{1}^{n+1} T_{F}=0$ and $T=\Delta^{n} T^{\prime}$ is equivalent 
to $T_{F}=\Delta_{1}^{n} T_{F}^{\prime}$ with $T_{F}^{\prime} \in L^{n-1}\left(\mathfrak{A}, \mathfrak{X}_{L}^{*, A}\right)_{F}$. Hence $H^{n}\left(\mathfrak{A}, \mathfrak{X}_{L}^{*, A}\right)=0$ is equivalent to $H^{n}\left(\mathfrak{A}, \mathfrak{X}_{L}^{*, A}\right)_{F}=0$ which proves the theorem. The proof is similar for $H^{n}\left(\mathfrak{A}, \mathfrak{X}_{R}^{*, A}\right), H^{n}\left(\mathfrak{A}, \mathfrak{X}^{*, A}\right)$ and $H^{n}\left(\mathfrak{A}, \mathfrak{X}^{*, A^{\prime \prime}}\right)$.

THEOREM 6. Let $p$ be a positive integer. Then

$$
\left\{\begin{array}{l}
H_{n+p}(\mathfrak{A}, \mathfrak{X}) \sim H_{n}\left(\mathfrak{A}, L_{p}(\mathfrak{A}, \mathfrak{X})\right) \\
H^{n+p}\left(\mathfrak{A}, \mathfrak{X}_{L}^{* \cdot A}\right) \sim H^{n}\left(\mathfrak{A}, L^{p}\left(\mathfrak{A}, \mathfrak{X}_{L}^{*, A}\right)\right)
\end{array}\right.
$$

and equivalent formulas for $\mathfrak{X}_{R}^{*, A}, \mathfrak{X}^{*, A}, \mathfrak{X}^{*, A^{\prime \prime}}$.

Proof. This is the Hochschild's method for the reduction of dimension [14] [15]. It consists in defining the natural isometry $\tau_{n}$ from $L_{n+p}(\mathfrak{A}, \mathfrak{X})$ onto $L_{n}\left(\mathfrak{A}, L_{p}(\mathfrak{A}, \mathfrak{X})\right)$ thanks to the associativity of the tensor product, the action of $\mathfrak{A}$ onto $L_{p}(\mathfrak{A}, \mathfrak{X})$ by

$$
\begin{aligned}
f \circ\left(f_{1} \otimes\right. & \left.\cdots \otimes f_{p} \otimes h\right)=f * f_{1} \otimes \cdots \otimes f_{p} \otimes h \\
= & \sum_{i=1}^{n-1}(-1)^{i} f \otimes f_{1} \otimes \cdots \otimes f_{i} * f_{i+1} \otimes \cdots \otimes f_{p} \otimes h \\
& +(-1)^{n} f \otimes f_{1} \otimes \cdots \otimes f_{p-1} \otimes f \circ h \\
\left(f_{1} \otimes\right. & \left.\cdots \otimes f_{p} \otimes h\right) \circ f=f_{1} \otimes \cdots \otimes f_{p} \otimes h
\end{aligned}
$$

and to notice that $\tau_{n-1} D_{n+p}=D_{n}^{\prime} \tau_{n}$ if $D_{n}^{\prime}$ denotes the equivalent of $D_{n}$ on $L_{n}\left(\mathfrak{A}, L_{p}(\mathfrak{A}, \mathfrak{X})\right)$.

In the same way, one can define an isometry $\tau^{n}$ from $L^{n+p}(\mathfrak{A}$, $\left.\mathfrak{X}_{L}^{*, A}\right)$ onto $L^{n}\left(\mathfrak{A}, L^{p}\left(\mathfrak{A}, \mathfrak{X}_{L}^{*, A}\right)\right)$ by

$$
\left[\left(\tau^{n} T\right)\left(f_{1} \otimes \cdots \otimes f_{n}\right)\right]\left(f_{n+1} \otimes \cdots \otimes f_{n+p}\right)=T\left(f_{1} \otimes \cdots \otimes f_{n+p}\right)
$$

and the "nondual" action of $\mathfrak{A}$ onto $L^{p}\left(\mathfrak{A}, \mathfrak{X}_{L}^{*, A}\right)$ by

$$
\begin{aligned}
& (f \cdot T)\left(f_{1} \otimes \cdots \otimes f_{p}\right)=f * T\left(f_{1} \otimes \cdots \otimes f_{p}\right) \\
& (T \cdot f)\left(f_{1} \otimes \cdots \otimes f_{p}\right)=T\left(f * f_{1} \otimes \cdots \otimes f_{p}\right) \\
& \quad+\sum_{i=1}^{n-1}(-1)^{i} T\left(f \otimes f_{1} \otimes \cdots \otimes f_{i} * f_{i+1} \otimes \cdots \otimes f_{p}\right) \\
& \quad+(-1)^{n} T\left(f \otimes f_{1} \otimes \cdots \otimes f_{p}\right) .
\end{aligned}
$$

A "dual" action could be defined according to $f \circ T=T$ and $T \circ f=$ T.f.

We close this paragraph by giving an example of $T \in L^{n}\left(\mathfrak{A}, \mathfrak{X}_{L}^{*, A}\right)$ in the "nondual" case. Let $\left(k_{i}\right), i=1, \cdots, n$ be a family of functions in $L^{\infty}(G),\left(F_{i}\right), i=1, \cdots, n$ a family of continuous linear forms in the dual $A^{\prime}$ of $A$, and $\mu \in \mathfrak{X}_{L}^{*}, A$. Let us define

$$
T=F_{1} \circ k_{1} \otimes \cdots \otimes F_{n} \circ k_{n}
$$


by

$$
\left\langle T, f_{1} \otimes \cdots \otimes f_{n}\right\rangle=F_{1}\left(\left\langle k_{1}, f_{1}\right\rangle\right) F_{2}\left(\left\langle k_{2}, f_{2}\right\rangle\right) \cdots F_{n}\left(\left\langle k_{n}, f_{n}\right\rangle\right)
$$

where

$$
\left\langle k_{i}, f_{i}\right\rangle=\int k_{i}(g) f_{i}(g) d g \in A .
$$

It is easy to check that $T$ has the desired properties for being an element of $L^{n}\left(\mathfrak{A}, \mathfrak{X}_{L^{*}}^{*}\right)$. Considering $f_{1} \otimes \cdots \otimes f_{n}$ as an element of $L_{1}\left(G^{n}, A^{\hat{\otimes}^{*} n}\right)(37), T$ is a function on $G^{n}$ with values in $\mathscr{L}\left(A^{\hat{\otimes}^{*}}, \mathfrak{X}_{L}^{*, A}\right)$ such that $\left\|T\left(g_{1}, \cdots, g_{n}\right)\right\|$ is in $L^{\infty}\left(G^{n}\right)$. It is the purpose of the next paragraph to prove that, under some hypothesis on $A$, any $T$ can be represented by a function having these properties and conversely.

5. A Riesz representation theorem for the elements of $L^{n}(\mathfrak{A}$, $\left.\mathfrak{X}_{R}^{*, A}\right), L^{n}\left(\mathfrak{A}, \mathfrak{X}_{L}^{*, A}\right)$, or $L^{n}\left(\mathfrak{A}, \mathfrak{X}^{*, A^{\prime \prime}}\right)$. In this section, we will restrict ourself to the case when $A=Z^{\prime}$, the dual of some Banach space $Z$, or when $A$ is a $C^{*}$-algebra (if $A$ is both, it is a Von Neumann algebra), and to the "nondual" structure. It could be posisible to adapt this paragraph to the "dual" one. We will denote by $\bar{a}$ and $\bar{g}$ an element of $A^{\hat{\otimes} n}$ and $G^{n}$ respectively.

We begin by recalling a theorem which asserts that any $T$ can be represented by a measure:

THEOREM 5. If $A$ is a Banach-*-algebra, there exists an isomorphism $T \leftrightarrow \mu_{T}$ between $L^{n}\left(\mathfrak{A}, \mathfrak{X}_{L}^{*, A}\right)$ (resp. $L^{n}\left(\mathfrak{A}, \mathfrak{X}_{R}^{*}{ }^{A}\right), L^{n}\left(\mathfrak{A}, \mathfrak{X}^{*, A^{\prime \prime}}\right)$ ) and the space of vector measures on $G^{n}$ with finite variation and with value in $\mathscr{L}\left(A^{\hat{\otimes}^{\hat{\otimes}}}, \mathfrak{X}_{L}^{*, A}\right)\left(\right.$ resp. $\left.\mathscr{L}\left(A^{\hat{\otimes}^{n}}, \mathfrak{X}_{R}^{*, A}\right), \mathscr{L}\left(A^{\hat{\otimes}^{*} n}, \mathfrak{X}^{*, A^{\prime \prime}}\right)\right)$ such that $\left|\mu_{T}\right|=k(\bar{g}) d|\bar{g}|$ with $h \in L^{\infty}\left(G^{n}\right), k \geqq 0$, where $d|\bar{g}|$ means the absolute value of the Haar measure on $G^{n}$, given by

$$
T\left(f_{1} \otimes \cdots \otimes f_{n}\right)=\int f_{1}\left(g_{1}\right) \cdots f_{n}\left(g_{n}\right) d \mu_{T}\left(g_{1}, \cdots, g_{n}\right)
$$

with

$$
\|T\|=\|k\|_{\infty}
$$

Proof. See ([7], 13, no. 3, Theorem 1, Corollary 2).

We are now going to prove that $\mu_{T}$ can be represented by a function, with the help of the following generalization of LebesgueNikodym's theorem. 
Proposition 8. Let $\nu$ be a regular Borel scalar measure on $G^{n}$, ㅅ a measure on $G^{n}$ with value in the Banach-*-algebra $A=Z^{\prime}$ (resp. in the $C$ *-algebra $A$ ), with finite variation $|\mu|$, absolutely continuous with respect to $\nu$ (i.e., $|\mu|$ is absolutely continuous with respect to $|\nu|$ in the usual sense). Then there exists a function $V_{\mu}$ on $G^{n}$ with value in $A$ (resp. in $A^{\prime \prime}$ ) such that:

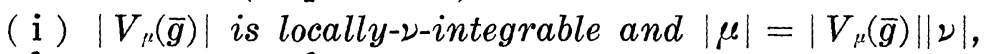
i.e.: $\int \rho(\bar{g}) d|\mu|(\bar{g})=\int \rho(\bar{g})\left|V_{\mu}(\bar{g})\right| d|\nu|(\bar{g}), \varphi \in L^{1}\left(G^{n},|\mu|\right)$, (ii) $\left\langle\int F(\bar{g}) d \mu(\bar{g}), z\right\rangle=\int\left\langle V_{\mu}(\bar{g}) F(\bar{g}), z\right\rangle d \nu(\bar{g}), F \in L^{1}\left(G^{n}, A, \mu\right)$ for any $z \in Z$ (resp. $z \in A^{\prime}$ ),

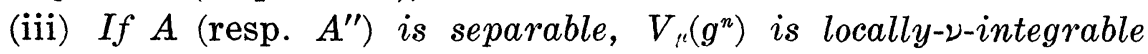
and

$$
\int F(\bar{g}) d \mu(\bar{g})=\int V_{\mu}(\bar{g}) F(\bar{g}) d \nu(\bar{g}),
$$

(iv) If $Z$ (resp. $A^{\prime}$ ) is separable, then $V_{" \prime}$ is unique.

Proof. See ([7], §13, $n^{\circ} 4$, Theorem 5).

Theorem 6. Let $A=Z^{\prime}, T \in L^{n}\left(\mathfrak{A}, \mathfrak{X}_{L}^{*, A}\right)$ (resp. $L^{n}\left(\mathfrak{A}, \mathfrak{X}_{R}^{*, A}\right)$ ) and $\mu_{T}$ the corresponding measure. There exists a function $V_{T}$ on $G^{n}$ with values in $\mathscr{C}\left(A^{\hat{\otimes} n}, \mathfrak{X}_{L}^{*}\right)$ (resp. $\mathscr{L}^{n}\left(A^{\hat{\otimes} n}, \mathfrak{X}_{R}^{*, A}\right)$ such that and

(i ) $\left\|V_{T}(\bar{g})\right\|=k(\bar{g}) \in L^{\infty}\left(G^{n}\right)$, where $k$ is defined in Theorem 5

$$
\int \varphi(\bar{g}) d\left|\mu_{T}\right|(\bar{g})=\int \rho(\bar{g})|| V_{T}(\bar{g}) \| d|\bar{g}|, \varphi \in L^{1}\left(G^{n},\left|\mu_{T}\right|\right)
$$

with $\|T\|=\|k\|_{\infty}=\|\| V_{T}(\bar{g})\|\|_{\infty}$.

(ii) $\left\langle V_{T}(\bar{g}) \Phi(\bar{g}), h\right\rangle$ is integrable, where $h \in \mathfrak{X}$ and $\Phi \in L^{1}\left(G^{n}, A^{\hat{\otimes}^{n}}\right)$ and

$$
\langle T(\Phi), h\rangle=\left\langle\int \Phi(\bar{g}) d \mu_{T}(\bar{g}), h\right\rangle=\int\left\langle V_{T}(\bar{g}) \Phi(\bar{g}), h\right\rangle d \bar{g} .
$$

(iii) If $A$ (and $\mathfrak{X}$ ) is separable, then $V_{T}$ is unique.

Proof. Let $B \in B\left(G^{n}\right)$ a Borel subset of $G^{n}$ and $\mu_{\frac{T}{a}}^{T}$ the measure on $G^{n}$ with value in $A$ defined by

$$
\mu_{\frac{T}{a}}^{T}(B)=\left\langle\mu_{T}(B) \bar{a}, h\right\rangle .
$$

It is a measure with finite variation and absolutely continuous with respect to $d \bar{g}$ because of

$$
\left|\mu_{\bar{a}, h}^{T}\right| \leqq|\bar{a}|\left|h\left\|_{\infty}\left|\mu_{T}\right|=|\bar{a}|\right\| h \|_{\infty} k(\bar{g}) d\right| \bar{g} \mid .
$$


Hence, by Proposition 8, there exists a function $G_{\bar{a}, h}^{T}$ on $G^{n}$ with values in $A$ such that

$$
\mu_{\bar{a}, h}^{T}=G_{\bar{a}, h}^{T} d \bar{g} \text { and }\left|\mu_{\bar{a}, h}^{T}\right|=\left|G_{\bar{a}, h}^{T}\right| d|\bar{g}| \leqq|\bar{a}|\|h\|_{\infty} k(\bar{g}) d|\bar{g}| .
$$

Let $G_{\bar{a}}^{T}(\bar{g})$ be the correspondence $h \in \mathfrak{X} \rightarrow G_{\bar{a}, h}^{T}(\bar{g})$. It is $A$-right linear and if $\left(h_{i}\right)$ is a finite family of functions in $K(G, A)$ with support $h_{i} \cap$ support $h_{j}=\varnothing$ and $\left\|\Sigma h_{i}\right\|_{\infty} \leqq 1$, then, for any $B \in B\left(G^{n}\right)$,

$$
\begin{aligned}
& \int_{B} \sum_{i}\left|G_{\bar{a}, h_{i}}^{T}(\bar{g})\right| d\left|\bar{g}=\sum_{i}\right| \mu_{\bar{a}, h_{i}}^{T}\left|(B)=\sup _{j} \sum_{i}\right| \mu_{\bar{a}, h_{i}}^{T}\left(B_{j}\right) \mid \\
& \quad=\sup _{j} \sum_{i}\left|\left\langle\mu_{T}\left(B_{j}\right) \bar{a}, h_{i}\right\rangle\right| \leqq \sup _{j}\left\|\mu_{T}\left(B_{j}\right) \bar{a}\right\|_{1} \leqq \sup _{j}\left\|\mu_{T}\left(B_{j}\right)\right\||\bar{a}| \\
& \quad=\left|\mu_{T}\right|(B)|\bar{a}|=\left(\int_{B} k(\bar{g}) d|\bar{g}|\right)|\bar{a}|
\end{aligned}
$$

from which we deduce that

$$
\sum_{i}\left|G_{\bar{a}, h_{i}}^{T}(\bar{g})\right| \leqq k(\bar{g})|\bar{a}|
$$

almost everywhere or, else, ||$\left|G_{\bar{a}}^{T}(\bar{g})\right| \||k(\bar{g})| \bar{a} \mid$ almost everywhere.

By modifying it on set of measure zero if necessary, we have $G \bar{T}(\bar{g}) \in \mathfrak{X}_{L}^{*}, A$ for any $\bar{g} \in G^{n}$ and

$$
\left\|G_{\bar{a}}^{T}(\bar{g})\right\|_{1} \leqq k(\bar{g})|\bar{g}| \text {. }
$$

If $V_{T}(\bar{g})$ is the correspondence $\bar{a} \rightarrow G_{\bar{a}}(\bar{a})$, then

$$
V_{T}(\bar{g}) \in \mathscr{L}\left(\boldsymbol{A}^{\hat{\otimes}^{n}}, \mathfrak{X}_{L}^{*, 1}\right),\left\|V_{T}(\bar{g})\right\| \leqq k(\bar{g}) \text {, and }\left\langle V_{T}(\bar{g}) \bar{a}, h\right\rangle=G_{\bar{a}, h}^{T^{\prime}}(\bar{g}) \text {. }
$$

For any step function $\Phi^{\prime}$ on $G^{n}$ with values in $A^{\hat{\otimes} n}$, we have

$$
\left\langle T\left(\Phi^{\prime}\right), h\right\rangle=\left\langle\int \Phi^{\prime}(\bar{g}) d \mu_{T}(\bar{g}), h\right\rangle=\int\left\langle V_{T}(\bar{g}) \Phi^{\prime}(\bar{g}), h\right\rangle d \bar{g}
$$

so that, if $\Phi_{i}^{\prime}$ is a sequence of such step functions converging to $\Phi$ in $L^{1}\left(G^{n}, A^{\hat{\otimes} n}\right)$, the Cauchy sequence $\left\langle V_{T}(\bar{g}) \Phi_{i}^{\prime}(\bar{g}), h\right\rangle$ converges to $\left\langle V_{T}(\bar{g}) \Phi(\bar{g}), h\right\rangle$ in $L^{1}\left(G^{n}, A\right)$ while $\int \Phi_{i}^{\prime}(\bar{g}) d \mu(\bar{g})$ converges to $\int \Phi(\bar{g}) d \mu(\bar{g})$ and (ii) is proved.

The inequality $\left\|V_{T}(\bar{g})\right\| \leqq k(\bar{g})$ shows that $\left\|V_{T}(\bar{g})\right\| \in L^{\infty}\left(G^{n}\right)$ and so is locally integrable. Then the inequality

$$
\left\langle\mu_{T}(B) \bar{a}, h\right\rangle \leqq \int_{B}\left|\left\langle V_{T}(\bar{g}) \bar{a}, h\right\rangle\right| d|\bar{g}|
$$

implies that

$$
\left\|\mu_{T}(B)\right\| \leqq \int_{B}\left\|V_{T}(\bar{g})\right\| d|\bar{g}|
$$


and

$$
\left|\mu_{T}\right|(B) \leqq \int_{B} \| V_{T}(\bar{g})|| d|\bar{g}|
$$

because $\left|\mu_{T}\right|$ is the least positive measure such that $\left\|\mu_{T}(B)\right\| \leqq$ $\left|\mu_{T}(B)\right|$. Hence

$$
\int_{B} k(\bar{g}) d|\bar{g}| \leqq \int_{B}|| V_{T}(\bar{g}) \| d|\bar{g}| \leqq \int k(\bar{g}) d \mid \bar{g} ;
$$

which proves (i).

If $A$ is separable, $X$ is separable too by countability of $G$. If $V_{T}^{\prime}$ is another function representing $\mu_{T}$,

$$
\int_{B}\left\langle\left(V_{T}(\bar{g})-V_{T}^{\prime}(\bar{g})\right) \bar{a}, h\right\rangle d \bar{g}=0
$$

for any Borel $B$ in $B\left(G^{n}\right)$, any $\bar{a}$ in $A^{\hat{\otimes}^{n}}$ and any $h$ in $\mathfrak{X}$. So $\left.\left\langle V_{T}(\bar{g})-V_{T}^{\prime}(\bar{g})\right) \bar{a}, h\right\rangle=0$ almost everywhere. Taking $\bar{a}$ and $h$ into countable dense subsets we conclude that $V_{T}(\bar{g})=V_{T}^{\prime}(\bar{g})$ almost everywhere.

Next theorem is a converse of the preceding one.

THEOREM 7. Let $A$ be a Banach-*-algebra and $V$ a function on $G^{n}$ with values in $\mathscr{L}\left(A^{\hat{\otimes} n}, \mathfrak{X}_{L}^{*, A}\right)$ (resp. $\mathscr{L}\left(A^{\hat{\otimes} n}, \mathfrak{X}_{R}^{*}{ }^{*}\right)$ ) such that $\|V(\bar{g})\| \in L^{\infty}\left(G^{n}\right)$ and $\langle V(\bar{g}) \bar{a}, h\rangle$ is measurable for any $\bar{a} \in A^{\hat{\otimes}^{n}}$ and $h \in \mathfrak{X}$. There exists a continuous linear map $T$ from $L^{1}\left(G^{n}, A^{\hat{\otimes}^{n}}\right)$ to $\mathfrak{X}_{L}^{*, A}\left(\right.$ resp. $\left.\mathfrak{X}_{R}^{*, A}\right)$ such that

$$
\langle T(\Phi), h\rangle=\int\langle V(\bar{g}) \Phi(\bar{g}), h\rangle d \bar{g}, \Phi \in L^{1}\left(G^{n}, A^{\hat{\otimes}}\right) .
$$

If $A=Z^{\prime}$, let $V_{T}$ and $k$ the corresponding functions (Theorems 6 and 5). Then

$$
k(\bar{g}) \leqq\|V(\bar{g})\| \text { a.e., and }\|T\| \leqq\|\| V(\bar{g})\|\|_{\infty} \text {. }
$$

If $A$ (and $\mathfrak{X}$ ) is separable, then $k(\bar{g})=\|V(\bar{g})\|$ a.e., $V=V_{T}$ and $\|T\|=\|\| V(\bar{g})\|\|_{\infty}$.

Proof. As $|\langle V(\bar{g}) \Phi(\bar{g}), h\rangle| \leqq\|V(\bar{g})\||\Phi(\bar{g})|\|h\|_{\infty}$ and

$$
\| V(\bar{g})|||\Phi(\bar{g})| \in L^{1}\left(G^{n}\right),|\langle V(\bar{g}) \Phi(\bar{g}), h\rangle|
$$

is integrable. Let $T_{h}(\Phi)=\int\langle V(\bar{g}) \Phi(\bar{g}), h\rangle d \bar{g}$ : the correspondence $h \rightarrow$ $T_{h}(\Phi)$, denoted $T(\Phi)$, is $A$-right linear and such that, if $\left(h_{i}\right)$ is a finite family of functions in $K(G, A)$ with support $h_{i} \cap$ support 


$$
\begin{aligned}
& h_{j}=\varnothing \text { and }\left\|\sum_{i} h_{i}\right\|_{\infty} \leqq 1 \text {, then } \\
& \qquad \begin{aligned}
\sum_{i}\left|T_{h_{i}}(\Phi)\right| & \leqq \int \sum_{i}\left|\left\langle V(\bar{g}) \Phi(\bar{g}), h_{i}\right\rangle\right| d|\bar{g}| \leqq \int\|V(\bar{g}) \Phi(\bar{g})\|_{1} d|\bar{g}| \\
& \leqq \int\|V(\bar{g})\||\Phi(\bar{g})| d|\bar{g}| \leqq\|\| V(\bar{g})\|\|_{\infty}\|\Phi\|_{1} .
\end{aligned}
\end{aligned}
$$

Hence $T(\Phi) \in \mathfrak{X}_{L}^{*, A}$ and $\|T(\Phi)\|_{1} \leqq\|\| V(\bar{g})\|\|_{\infty}\|\Phi\|_{1}$. So the correspondence $\Phi \rightarrow T(\Phi)$, denoted $T$, is linear and continuous with $\|T\| \leqq\|\| V(\bar{g})\|\| \|_{\infty}:$ hence $T \in L^{n}\left(\mathfrak{A}, \mathfrak{X}_{L}^{* \cdot A}\right)$ and

$$
\langle T(\Phi), h\rangle=\int\langle V(\bar{g}) \Phi(\bar{g}), h\rangle d \bar{g}=\left\langle\int \Phi(\bar{g}) d \mu_{T}(\bar{g}), h\right\rangle
$$

if $\mu_{T}$ is the corresponding measure according to Theorem 5 with $\left|\mu_{T}\right|=k(\bar{g}) d|\bar{g}|, k \in L^{\infty}\left(G^{n}\right)$. So, in particular,

$$
\left|\mu_{T}\right|(B) \leqq \int_{B}\|V(\bar{g})\| d|\bar{g}| \text { and }\left|\mu_{T}\right| \leqq\|V(\bar{g})\| d|\bar{g}| \text {, i.e., } k(\bar{g}) \leqq\|V(\bar{g})\|
$$

almost everywhere. Moreover, if $V_{T}$ is the function corresponding to $T$ by Theorem 6 ,

$$
\int\langle V(\bar{g}) \Phi(\bar{g}), h\rangle d \bar{g}=\int\left\langle V_{T}(\bar{g}) \Phi(\bar{g}), h\right\rangle d \bar{g}
$$

and, in particular,

$$
\langle V(\bar{g}) \bar{a}, h\rangle=\left\langle V_{T}(\bar{g}) \bar{a}, h\right\rangle \text { a.e. , }
$$

which ends the proof of the theorem if $A$ (and $\mathfrak{X}$ ) is separable.

REMARK. If the hypothesis $A=Z^{\prime}$ is replaced by $A$ is a $C^{*}$ algebra, then $V_{T}$ is, in any case, a function on $G^{n}$ with values in $\mathscr{C}\left(A^{\hat{\otimes} n}, \mathfrak{X}^{*, A^{\prime \prime}}\right)$ : the proof of Theorem 6 is similar but the difference comes from Radon-Nikodym's theorem (Proposition 8). But then Theorem 7 associates to $V$ an element of $L^{n}\left(\mathfrak{A}, \mathfrak{X}^{*} A^{\prime \prime}\right)$ and so is no longer the converse of Theorem 6 . The symmetry is restored if we start with $L^{n}\left(\mathfrak{A}, \mathfrak{X}^{*, A^{\prime \prime}}\right)$ : if $A$ (and $\mathfrak{X}$ ) is separable, this space is isometrically isomorphic to the space of functions $V$ on $G^{n}$ with values in $\mathscr{L}\left(A^{\hat{\otimes}_{n}}, \mathfrak{X}^{*, A^{\prime \prime}}\right)$ such that $\|V(\bar{g})\| \in L^{\infty}\left(G^{n}\right)$ and $\langle V(\bar{g}) \Phi(\bar{g}), h\rangle$ is measurable for any $\Phi \in L^{1}\left(G^{n}, A^{\hat{\otimes} n}\right)$ and $h \in \mathfrak{X}$.

6. Extension from $\mathfrak{A}$ to $\mathfrak{X}_{L}^{*, A}, \mathfrak{X}_{R}^{*, A}$ or $\mathfrak{X}^{*, A}$. By Propositions 5 and 6 , it is possible to define $L_{n}\left(\mathfrak{X}_{L}^{*, A}, \mathfrak{X}\right)$ and $L^{n}\left(\mathfrak{X}_{L}^{*, A}, \mathfrak{X}_{L}^{*, A}\right)$, or $L^{n}\left(\mathfrak{X}_{L}^{*, A}, \mathfrak{X}^{*, A^{\prime \prime}}\right)$ if $A$ is a $C^{*}$-algebra, (and the same for $\mathfrak{X}_{R}^{*, A}$ or $\mathfrak{X}^{*, A}$ ) in the same way as we did for $\mathfrak{A}$. In this paragraph, we shall state the relation between these spaces and the corresponding ones 
for $\mathfrak{A}$, under the hypothesis: $A=Z^{\prime}$ or $A$ is a $C^{*}$-algebra.

THEOREM 8. Let $A=Z^{\prime}$ be a Banach-*-algebra. It is possible to extend $T \in L^{n}\left(\mathfrak{A}, \mathfrak{X}_{L}^{*, A}\right)$ (resp. $L^{n}\left(\mathfrak{A}, \mathfrak{X}_{R}^{*, A}\right), L^{n}\left(\mathfrak{A}, \mathfrak{X}^{*, A}\right)$ ) as an element $\widetilde{T}$ of $L^{n}\left(\mathfrak{X}_{L}^{*, A}, \mathfrak{X}_{L}^{*, A}\right)$ (resp. $\left.L^{n}\left(\mathfrak{X}_{R}^{*, A}, \mathfrak{X}_{R}^{*, A}\right), L^{n}\left(\mathfrak{X}^{*, A}, \mathfrak{X}^{*, A}\right)\right)$ with $\|T\|=\|\widetilde{T}\|$.

Proof. Let $\left(\mu_{i}\right), i=1, \cdots, n$ with $\mu_{i} \in \mathfrak{X}_{L}^{*, A}, h \in \mathfrak{X}$ and $\left\{e_{\alpha} \lambda_{\beta}\right\}_{\alpha \in I: \beta \in J}$ a (countable) approximate unit in 2 . The set of $\left\langle T\left(\mu_{1} * e_{\alpha_{1}} \lambda_{\beta_{1}}, \cdots\right.\right.$, $\left.\left.\mu_{n} * e_{\alpha_{n}} \lambda_{\beta_{n}}\right), h\right\rangle$ is bounded in $A$, uniformly with respect to $\alpha_{i}$ and $\beta_{i}$, and also in the bidual $A^{\prime \prime}$. So there exists a (nonunique) sequence of such elements converging weakly in the bidual $A^{\prime \prime}$ towards an element $\widetilde{T}\left(\mu_{1}, \cdots, \mu_{n}, h\right)$ which is evidently linear in $\mu_{1}, \cdots, \mu_{n}$. It is $A$-right linear in $h$ because, for any $F \in A^{\prime}$,

$$
\begin{aligned}
\operatorname{limit}_{i_{k}, j_{k}} & \left\langle\left\langle T\left(\mu_{1} * e_{i_{1}} \lambda_{j_{1}}, \cdots, \mu_{n} * e_{i_{n}} \lambda_{j_{n}}, h a\right), F\right\rangle\right. \\
& =\left\langle\widetilde{T}\left(\mu_{1}, \cdots, \mu_{n}, h a\right), F\right\rangle \\
& =\operatorname{limit}_{i_{k}, j_{k}}\left\langle T\left(\mu_{1} * e_{i_{1}} \lambda_{j_{1}}, \cdots, \mu_{n} * e_{i_{n}} \lambda_{j_{n}}, h\right) a, F\right\rangle \\
& =\operatorname{limit}_{i_{k}, j_{k}}\left\langle T\left(\mu_{1} * e_{i_{1}} \lambda_{j_{1}}, \cdots, \mu_{n} * e_{i_{n}} \lambda_{j_{n}}\right), a^{t} F\right\rangle \\
& =\left\langle\widetilde{T}\left(\mu_{1}, \cdots, \mu_{n}\right), a^{t} F\right\rangle=\left\langle\widetilde{T}\left(\mu_{1} \cdots \mu_{n}\right) a, F\right\rangle
\end{aligned}
$$

where the multiplication on the right of an element in the bidual $A^{\prime \prime}$ by an element in $A$ is defined through bitransposition [1], [5]. Moreover, as $A=Z^{\prime}$ (i.e., $\left.A^{\prime}=Z^{\prime \prime} \supset Z\right), \widetilde{T}\left(\mu_{1}, \cdots, \mu_{n}, h\right)$ is in fact in $A \subset A^{\prime \prime}$, as we can see computing the limit for $F=z \in Z \subset Z^{\prime \prime}$, and the right multiplication by a coincide with the product in $A$.

Now, given $\varepsilon>0$ and $z \in Z$, there exists $i_{0, k}$ and $j_{0, k}$ such that, if $i_{k}>i_{0, k}$ and $j_{k}>j_{0, k}$,

$$
\left|\left\langle\widetilde{T}\left(\mu_{1}, \cdots, \mu_{n}, h\right), z\right\rangle\right| \leqq\left|\left\langle T\left(\mu_{1} * e_{i_{1}} \lambda_{j_{1}}, \cdots, \mu_{n} * e_{i_{n}} \lambda_{j_{n}}\right), h\right\rangle, z\right\rangle \mid+\varepsilon .
$$

So, if $\left(h_{l}\right), l=1, \cdots, m$ is a finite family in $K(G, A)$ with support $h_{l} \cap$ support $h_{l^{\prime}}=\varnothing$ for $l \neq l^{\prime}$ and $\left\|\sum_{l} h_{l}\right\|_{\infty} \leqq 1$, then

$$
\begin{aligned}
\sum_{l}\left|\left\langle\widetilde{T}\left(\mu_{1}, \cdots, \mu_{n}, h_{l}\right), z\right\rangle\right| \leqq \sum_{l}\left\langle\left\langle T\left(\mu_{1} * e_{i_{1}} \lambda_{j_{1}}, \cdots, \mu_{n} * e_{i_{n}} \lambda_{j_{n}}\right), h_{l}\right\rangle, z\right\rangle \mid+m \varepsilon \\
\quad \leqq\|z\| \sum_{l}\left|\left\langle T\left(\mu_{1} * e_{i_{1}} \lambda_{j_{1}}, \cdots, \mu_{n} * e_{i_{n}} \lambda_{j_{n}}\right), h_{l}\right\rangle\right|+m \varepsilon \\
\quad \leqq\|z\|\left\|T\left(\mu_{1} * e_{i_{1}} \lambda_{j_{1}}, \cdots \mu_{n} * e_{i_{n}} \lambda_{j_{n}}\right)\right\|_{1}+m \varepsilon \\
\quad \leqq\|z\|\|T\|\left\|\mu_{1}\right\|_{1} \cdots\left\|\mu_{n}\right\|_{1}+m \varepsilon .
\end{aligned}
$$

Consequently,

$$
\sum_{l}\left|\widetilde{T}\left(\mu_{1}, \cdots, \mu, h_{l}\right)\right| \leqq\|T\|\left\|\mu_{1}\right\| \cdots\left\|\mu_{n}\right\|
$$

which proves that $\widetilde{T} \in L^{n}\left(\mathfrak{X}_{L}^{*}, A, \mathfrak{X}_{L}^{*}, A\right)$ with $\|\widetilde{T}\| \leqq\|T\|$. But, if $\left(f_{i}\right)$, 
$i=1, \cdots, n$ is a finite family in $\mathfrak{A}$,

$$
\begin{gathered}
\operatorname{limit}_{i_{k}, j_{k}}\left\langle T\left(f_{1} * e_{i_{1}} \lambda_{j_{1}}, \cdots, f_{n} * e_{i_{n}} \lambda_{j_{n}}\right), h\right\rangle=\widetilde{T}\left(f_{1}, \cdots, f_{n}, h\right) \\
=\left\langle T\left(f_{1}, \cdots, f_{n}\right), h\right\rangle
\end{gathered}
$$

where the convergence is even in norm. Hence $\widetilde{T}$ restricted to $\mathfrak{A}$ is $T$ and $\|\widetilde{T}\|=\|T\|$ because

$$
\|\widetilde{T}\|=\sup _{\left\|\mu_{i}\right\|_{1} \leq 1}\left\|\widetilde{T}\left(\mu_{1}, \cdots, \mu_{n}\right)\right\|_{1} \geqq \sup _{\left\|f_{i}\right\|_{1} \leq 1}\left\|T\left(f_{1}, \cdots, f_{n}\right)\right\|_{1}=\|T\| .
$$

REMARK. If the hypothesis $A=Z^{\prime}$ is replaced by $A$ is a $C^{*}$ algebra, the preceding proof shows that $\widetilde{T} \in L^{n}\left(\mathfrak{X}_{L}^{*, 4}, \mathfrak{X}^{*, A^{\prime \prime}}\right)$. So, once again (see remark at the end of the preceding paragraph) we see that, in the case of a $C^{*}$-algebra, $L^{n}\left(\mathfrak{A}, \mathfrak{X}^{*, A^{\prime \prime}}\right)$ is a more natural object than $L^{n}\left(\mathfrak{A}, \mathfrak{X}_{L}^{*, A}\right)$.

Thanks to Theorem 8 , we can give a meaning to the function $\widetilde{T}(\bar{g})$ on $G^{n}$ by $\widetilde{T}(\bar{g})=\widetilde{T}\left(\delta_{g_{1}}, \cdots, \delta_{g_{n}}\right)$ if $\bar{g}=\left(g_{1}, \cdots, g_{n}\right) \in G^{n}$. We are going to see that this function is closely related to the function $V_{T}$ defined in Theorem 6 (see also the remark at the end of the preceding paragraph).

THEOREM 9. Let $A$ (and $\mathfrak{X}$ ) be separable and let $\lambda_{j}$ be a countable approximate unit in $L^{1}(G)$ such that $\lim _{j} \lambda_{j} * k=k$ almost everywhere for any $k \in L^{\infty}(G, A)$ or $L^{\infty}\left(G, A^{\prime \prime}\right)$. (This condition is discussed in ([12, Theorem 44. 18]). If $\bar{a}=\left(a_{1}, \cdots, a_{n}\right) \in A^{\hat{\otimes}^{n}}$ and $\bar{g}=$ $\left(g_{1}, \cdots, g_{n}\right) \in G^{n}$, then, with any of the two hypothesis on $A$,

$$
\widetilde{T}\left(a_{1} \delta_{g_{1}}, \cdots, a_{n} \delta_{g_{n}}\right)=V_{T}(\bar{g}) \bar{a}
$$

almost everywhere.

Proof. Let $\left(\varphi_{i}\right), i=1, \cdots, n$ with $\varphi_{i} \in L^{1}(G)$. For any $h \in \mathfrak{X}$ $\left\langle T\left(a_{1} \varphi_{1}, \cdots, a_{n} \varphi_{n}\right), h\right\rangle=\int \varphi_{1}\left(g_{1}\right) \cdots \varphi_{n}\left(g_{n}\right)\left\langle V_{T}(\bar{g}) \bar{a}, h\right\rangle d \bar{g}$ and $\left\langle V_{T}(\bar{g}) \bar{a}, h\right\rangle \epsilon$ $L^{\infty}\left(G^{n}, A\right)$ or $L^{\infty}\left(G^{n}, A^{\prime \prime}\right)$. In particular,

$$
\left.T\left(a_{1} \delta_{g_{1}} * \lambda_{j_{1}}, \cdots, a_{n} \delta_{g_{n}} * \lambda_{j_{n}}\right), h\right\rangle=\int \lambda_{j_{1}}\left(g_{1}^{-1} u_{1}\right) \cdots \lambda_{j_{n}}\left(g_{n}^{-1} u_{n}\right)\left\langle V_{T}(\bar{u}) \bar{a}, h\right\rangle d \bar{u} .
$$

By hypothesis on $\lambda_{j}$ and by successive applications of Lebesgue's dominated convergence theorem, we obtain

$$
\left\langle\widetilde{T}\left(a_{1} \delta_{g_{1}}, \cdots, a_{n} \delta_{g_{n}}\right), h\right\rangle=\left\langle V_{T}(\bar{g}) \bar{a}, h\right\rangle
$$

almost everywhere and, by separability of $\mathfrak{X}$,

$$
\widetilde{T}\left(a_{1} \delta_{g_{1}}, \cdots, a_{n} \delta_{g_{n}}\right)=V_{T}(\bar{g}) \bar{a}, \text { a.e. }
$$


We then have the following formulas

$$
\begin{gathered}
\langle T(\bar{a} \Phi), h\rangle=\int \Phi(\bar{g})\left\langle\widetilde{T}\left(a_{1} \delta_{g_{1}}, \cdots, a_{n} \delta_{g_{n}}\right), h\right\rangle d \bar{g}, \Phi \in L^{1}\left(G^{n}\right), \\
\bar{a}=\left(a_{1}, \cdots, a_{n}\right), \bar{g}=\left(g_{1}, \cdots, g_{n}\right) \\
\left\langle T\left(a_{1} \varphi_{1} * a_{2} \varphi_{2}\right), h\right\rangle=\int \varphi_{1}\left(g_{1}\right) \varphi_{2}\left(g_{2}\right)\left\langle\widetilde{T}\left(a_{1} \sigma\left(g_{1}\right) a_{2} \delta_{g_{1} g_{2}}\right), h\right\rangle d g_{1} d g_{2} \\
=\int \varphi_{1}\left(g_{1}\right) \varphi_{2}\left(g_{2}\right)\left\langle V_{T}\left(g_{1}, g_{2}\right) a_{1} \sigma\left(g_{1}\right) a_{2}, h\right\rangle d g_{1} d g_{2}, \varphi_{1}, \varphi_{2} \in L^{1}(G) .
\end{gathered}
$$

We can now determine the relation between $L^{n}\left(\mathfrak{A}, \mathfrak{X}_{L}^{*}, A\right)$ (resp. $\left.L^{n}\left(\mathfrak{A}, \mathfrak{X}^{*, A^{\prime \prime}}\right)\right)$ and $L^{n}\left(\mathfrak{X}_{L}^{*, A}, \mathfrak{X}_{L}^{*, A}\right)\left(\operatorname{resp} . L^{n}\left(\mathfrak{X}_{L}^{*, A}, \mathfrak{X}^{*, A^{\prime \prime}}\right)\right)$.

THEOREM 10. The restriction operation $\widetilde{T} \rightarrow T$ from $\mathfrak{X}_{L}^{*, A}$ to $\mathfrak{A}$ and $\Delta^{n}$ commute. This restriction induces a map from $L^{n}\left(\mathfrak{X}_{L}^{*, A}, \mathfrak{X}_{L}^{*, A}\right)$ (resp. $\left.L^{n}\left(\mathfrak{X}_{L}^{*, A}, \mathfrak{X}, A^{\prime \prime}\right)\right)$ to $L^{n}\left(\mathfrak{A}, \mathfrak{X}_{L}^{*, A}\right)$ (resp. $L^{n}\left(\mathfrak{A}, \mathfrak{X}^{*, A^{\prime \prime}}\right)$ ) such that $H^{n}\left(\mathfrak{X}_{L}^{*, A}, \mathfrak{X}_{L}^{*, A}\right)$ (resp. $\left.H^{n}\left(\mathfrak{X}_{L}^{*}, A, \mathfrak{X}^{*, A^{\prime \prime}}\right)\right)$ is isomorphic to $H^{n}\left(\mathfrak{A}, \mathfrak{X}_{L}^{*, A}\right)$ (resp. $\left.H^{n}\left(\mathfrak{A}, \mathfrak{X}^{*, A^{\prime \prime}}\right)\right)$.

Proof. The proof is by induction, identical to the corresponding one in ([15], Prop. 1.9 and Lemma 1.10), the proof of Lemma 1.10 being now achieved by noticing that $f * \mu_{L}=0$ for any $f \in \mathfrak{X}$ imply $\mu_{L}=0$.

7. Vector means. This paragraph collects and proves results, some of which will be useful in the sequel, about vector means, a natural extension of usual means on locally compact groups [10]. This notion appeared for the first time in [9].

We shall consider the following functional spaces:

$X_{1}=L^{\infty}(G, A)$;

$X_{2}=C B(G, A)$, the space of bounded continuous functions on $G$ with value in $A$;

$X_{3}=U C B_{r}^{o}(G, A)$, the space of bounded continuous functions $k$ on $G$ with value in $A$ with the property that, for any $\varepsilon>0$, there exists $\mathscr{Y}(e)$, neighborhood of $e$ in $G$, such that, for any $g \in \mathscr{V}(e)$, $\left|k(v)-\sigma(g) k\left(g^{-1} v\right)\right| \leqq \varepsilon$ for any $v \in G$;

$X_{4}=U C B_{r}(G, A)$, the space of bounded continuous functions $k$ on $G$ with value in $A$ with the property that, for any $\varepsilon>0$, there exists $\mathscr{V}(e)$, neighborhood of $e$ in $G$, such that, for any $g \in \mathscr{V}(e)$, $\left|k(v)-k\left(g^{-1} v\right)\right| \leqq \varepsilon$ for any $v \in G$;

$X_{5}=U C B_{l}(G, A)$, the space of bounded continuous functions $k$ on $G$ with value in $A$ with the property that, for any $\varepsilon>0$, there exists $\mathscr{C}(e)$, neighborhood of $e$ in $G$, such that, for any $g \in \mathscr{V}(e)$, $|k(v)-k(v g)| \leqq \varepsilon$ for any $v \in G$; 


$$
\begin{aligned}
& X_{6}=X_{3} \cap X_{5} ; \\
& X_{7}=X_{4} \cap X_{5} .
\end{aligned}
$$

Each of these spaces is a $C^{*}$-algebra for the usual product of functions, the uniform norm and the involution $k \rightarrow \bar{k}: \bar{k}(g)=k(g)^{*}$, and we have the following inclusions:

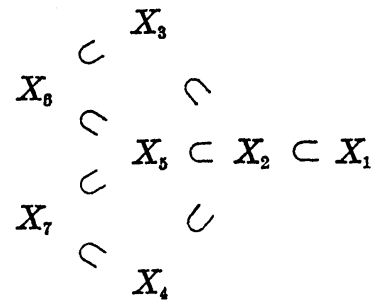

Definitions. An $A$-mean on $X_{i}$ is an $A$-linear positive continuous map on $X_{i}$ with value in $A$, with norm less than or equal to 1 . An $A$-mean on $X_{1}, X_{2}, X_{4}$ or $X_{7}$ is said left invariant if

$$
\left\langle M, k\left(g^{-1} .\right)\right\rangle=\langle M, k\rangle
$$

and topologically left invariant if (here * exceptionally means the usual convolution)

$$
\langle M, \varphi * k\rangle=\langle M, k\rangle \int \varphi(u) d u, \quad \varphi \in L^{1}(G) .
$$

An $A$-mean on $X_{1}, X_{2}, X_{3}$ or $X_{6}$ is said $\sigma$-left invariant if

$$
\left\langle M, \sigma(g) k\left(g^{-1} .\right)\right\rangle=\sigma(g)\langle M, k\rangle
$$

and topologically $\sigma$-left invariant if

$$
\left\langle M, \varphi^{*^{\sigma}} k\right\rangle=\int \varphi(u) \sigma(u)\langle M, k\rangle d u, \quad \varphi \in L^{1}(G) .
$$

An $A$-mean on $X_{1}, X_{2}, X_{5}$ or $X_{7}$ is said right invariant if

$$
\langle M, k(\cdot g)\rangle=\langle M, k\rangle
$$

and topologically right invariant if (here usual and $\sigma$-convolution coincide)

$$
\langle M, k * \widetilde{\varphi}\rangle=\langle M, k\rangle \int \varphi(u) d u, \varphi \in L^{1}(G), \widetilde{\varphi}(u)=\varphi\left(u^{-1}\right) .
$$

These definitions make sense thanks to the following lemmas:

Lemma 1. If $\varphi \in L^{1}(G)$ and $k \in L^{\infty}(G, A)$, then $\varphi * k$ (the usual convolution) is in $X_{4}$ and $k * \widetilde{\varphi}$ in $X_{5}$. If $k$ is in $X_{5}, \varphi * k$ is in $X_{7}$ 
and if $k$ is in $X_{4}, k * \widetilde{\varphi}$ is in $X_{7}$.

Proof.

$$
\begin{aligned}
\left|(\varphi * k)(v)-(\varphi * k)\left(g^{-1} v\right)\right| & \leqq \int\left|\varphi(v u)-\varphi\left(g^{-1} v u\right)\right|\left|k\left(u^{-1}\right)\right| d u \\
& \leqq\left\|\varphi-\varphi\left(g^{-1} \cdot\right)\right\|_{1}\|k\|_{\infty}
\end{aligned}
$$

and

$$
\begin{aligned}
|(k * \widetilde{\rho})(v)-(k * \widetilde{\varphi})(v g)| & \leqq \int|k(u)|\left|\varphi\left(v^{-1} u\right)-\varphi\left(g^{-1} v^{-1} u\right)\right| d u \\
& \leqq\left\|\varphi-\varphi\left(g^{-1} .\right)\right\|_{1}\|k\|_{\infty},
\end{aligned}
$$

and the conclusion comes from ([13], Theorem (20.4)).

If $k \in X_{5}$, it is enough to prove that $\varphi_{* k}$ is in $X_{5}$ but

$$
\begin{aligned}
|(\varphi * k)(v)-(\varphi * k)(v g)| & \leqq \int|\varphi(u)|\left|k\left(u^{-1} v\right)-k\left(u^{-1} v g\right)\right| d u \\
& \leqq\|\varphi\|_{1}\|k-k(. g)\|_{\infty} .
\end{aligned}
$$

If $k \in X_{4}$, it is enough to prove that $k * \widetilde{\varphi}$ is in $X_{4}$ but

$$
\begin{aligned}
\left|(k * \tilde{\varphi})(v)-(k * \widetilde{\varphi})\left(g^{-1} v\right)\right| & \leqq \int\left|k(v u)-k\left(g^{-1} v u\right)\right||\varphi(u)| d u \\
& \leqq\|\varphi\|_{1}\left\|k-k\left(g^{-1} \cdot\right)\right\|_{\infty} .
\end{aligned}
$$

LEMma 1 bis. If $\varphi \in L^{1}(G)$ and $k \in L^{\infty}(G, A)$, then $\varphi *^{\sigma} k \in X_{3}$ and $k *^{\circ} \widetilde{\varphi} \in X_{5}$. If $k \in X_{5}$ then $\varphi *^{\sigma} k \in X_{6}$ and if $k \in X_{3}$ then $k *^{\circ} \widetilde{\varphi}=k * \widetilde{\varphi} \in X_{6}$.

Proof.

$$
\begin{aligned}
& \left|\left(\varphi *^{\sigma} k\right)(v)-\sigma(g)(\varphi * k)\left(g^{-1} v\right)\right|=\mid \int \varphi(v u) \sigma(v u) k\left(u^{-1}\right) d u-\sigma(g) \\
& \quad \times \int \varphi\left(g^{-1} v u\right) \sigma\left(g^{-1} v u\right) k\left(u^{-1}\right) d u\left|\leqq \int\right| \varphi(v u)-\varphi\left(g^{-1} v u\right)|| \sigma(v u) k\left(u^{-1}\right) \mid d u \\
& \quad \leqq\left\|\varphi-\varphi\left(g^{-1} \cdot\right)\right\|_{1}\|k\|_{\infty}
\end{aligned}
$$

and $k *^{\sigma} \tilde{\varphi}=k * \tilde{\varphi}$ (see above).

If $k \in X_{5}$, it is enough to prove that $\varphi_{*} k \in X_{5}$ but

$$
\begin{aligned}
|(\varphi * k)(v)-(\varphi * k)(v g)| & \leqq \int|\varphi(u)|\left|\sigma(u) k\left(u^{-1} v\right)-\sigma(u) k\left(u^{-1} v g\right)\right| d u \\
& \leqq\|\varphi\|_{1}\|k-k(. g)\|_{\infty}
\end{aligned}
$$

and if $k \in X_{3}$ it is enough to prove that $k * \widetilde{\varphi}$ is in $X_{3}$, but

$$
\begin{aligned}
\left|(k * \widetilde{\varphi})(v)-\sigma(g)(k * \widetilde{\varphi})\left(g^{-1} v\right)\right| & \leqq \int\left|k(v u)-\sigma(g) k\left(g^{-1} v u\right)\right||\varphi(u)| d u \\
& \leqq\|\varphi\|_{1}\left\|k-\sigma(g) k\left(g^{-1} \cdot\right)\right\|_{\infty} .
\end{aligned}
$$


The relation between these different means and these different spaces comes from the following proposition.

Proposition 9. If there exists a left invariant $A$-mean, there exists a right invariant one on the corresponding space, and conversely.

Proof. Let us consider the transformation $k \rightarrow \tilde{k}: k(u)=k\left(u^{-1}\right)$. It changes $X_{1}, X_{2}, X_{4}$ or $X_{7}$ into $X_{1}, X_{2}, X_{5}$ or $X_{7}$ because if $k \in X_{4}$ then $\tilde{k}(v)-\widetilde{k}(v g)=k\left(v^{-1}\right)-k\left(g^{-1} v^{-1}\right)$. Let $\langle\bar{M}, \widetilde{k}\rangle=\langle M, k\rangle$. Then, if $M$ is left invariant, $\langle\bar{M}, \tilde{k}(. g)\rangle=\left\langle M, k\left(g^{-1}.\right)\right\rangle=\langle M, k\rangle=\langle\bar{M}, \widetilde{k}\rangle$.

THEOREM 11. Let $M$ a $\sigma$-left, left or right invariant A-mean on $X_{1}$ or $X_{2}$. Then $M$ restricted to $\mathfrak{X}=\mathscr{C}_{0}(G, A)$ is null if $G$ is non- compact.

Proof. Let $V$ be a relatively compact neighborhood of $e$ in $G$. By noncompacity of $G$, there is no finite sequence $\left(g_{i}\right)$ in $G$ such that $\left(g_{i} V\right)$ is a covering of $G$. So it is possible to choose an infinite sequence $\left(g_{i}\right)$ in $G$ such that $g_{n+1} \notin \bigcup_{i=1}^{n} g_{i} V$ for all integer $n$. Hence if $U$ is a symmetric relatively compact neighborhood of $e$ such that $U^{2} \subset V$, the sequence $\left(g_{i} U\right)$ is made of pairwise disjoint sets. Let $\widetilde{M}$ the canonical extension of $M$ to $\widetilde{X}_{1}$ or $\widetilde{X}_{2}$, the functional spaces corresponding to $\widetilde{A}$, the associative Banach-*-algebra with unit, defined according to $\langle M, a \varphi\rangle=a\langle\tilde{M} \cdot \varphi\rangle$ for any $a \in A$ and $\varphi$ any function with scalar values belonging to $\tilde{X}_{1}$ or $\tilde{X}_{2}$. Then $a\langle\tilde{M}$, $\left.\varphi\left(g^{-1}.\right)\right\rangle=\left\langle M, a \varphi\left(g^{-1}.\right)\right\rangle=\left\langle M, \sigma(g) \sigma\left(g^{-1}\right) a \varphi\left(g^{-1}.\right)\right\rangle=\sigma(g)\left\langle M, \sigma\left(g^{-1}\right) a \varphi\right\rangle=$ $a\langle\tilde{M}, \varphi\rangle$, i.e., $\left\langle\tilde{M}, \varphi\left(g^{-1}.\right)\right\rangle=\langle\tilde{M}, \varphi\rangle$. Let $\xi_{U} \in \widetilde{X}_{1}$ or $\widetilde{X}_{2}$ be the characteristic function of $U$ : as $\sum_{i=1}^{n} \xi_{g_{i} U} \leqq 1$, we have $\sum_{i=1}^{n}\left\langle\tilde{M}, \xi_{U}\right\rangle \leqq 1$, i.e., $\left\langle\widetilde{M}, \xi_{U}\right\rangle=0$. Let $h \in \mathfrak{X}$. Given $\varepsilon$, there exists a compact $K \subset G$ such that $|h(g)|<\varepsilon$ for $g \notin K$ and a finite sequence $\left(g_{j}^{\prime}\right)$ in $G$ such that $K \subset \cup_{j} g_{j}^{\prime} U$. So $|h(g)| \leqq\|h\| \sum_{j} \xi_{g_{j}^{\prime} U}+\varepsilon$ and $|\langle M, h\rangle| \leqq\langle\tilde{M}, \varepsilon\rangle=$ $\varepsilon\langle\tilde{M}, 1\rangle \leqq \varepsilon$. The proof is similar if $M$ is left or right invariant.

The relation between topological left or right invariance and left or right invariance is given by the two following theorems.

Theorem 12. A $\sigma$-left invariant $A$-mean is topologically $\sigma$-left invariant. A left (or right) invariant $A$-mean is topologically left (or right) invariant.

Proof. Let $\varphi \in L_{1}(G)$. Then, for $k \in X_{i}, i=1,2,3$, or 6 , $\int \varphi(u) \sigma(u)\left\langle M, \sigma(g) k\left(g^{-1} \cdot\right)\right\rangle d u=\left\langle M, \varphi * \sigma(g) k\left(g^{-1} \cdot\right)\right\rangle$ 


$$
\begin{aligned}
& =\left\langle M, \int \varphi(u) \sigma(u g) k\left(g^{-1} u^{-1} \cdot\right) d u\right\rangle \\
& =\left\langle M, \delta\left(g^{-1}\right) \int \varphi\left(u g^{-1}\right) \sigma(u) k\left(u^{-1} \cdot\right) d u\right\rangle \\
& =\left\langle M, \delta\left(g^{-1}\right) \varphi\left(. g^{-1}\right) * k\right\rangle=\delta\left(g^{-1}\right) \int \varphi\left(u g^{-1}\right) \sigma(u)\langle M, k\rangle d u \\
& =\int \varphi(u) \sigma(u) \sigma(g)\langle M, k\rangle d u .
\end{aligned}
$$

This being true for any $\varphi,\left\langle M, \sigma(g) k\left(g^{-1}.\right)\right\rangle=\sigma(g)\langle M, k\rangle$. The proof is similar for left and right invariance.

Theorem 13. A $\sigma$-left invariant $A$-mean on $X_{3}\left(\right.$ or $\left.X_{6}\right)$ is topologically $\sigma$-invariant. A left invariant $A$-mean on $X_{4}\left(\right.$ or $\left.X_{7}\right)$ is topologically invariant, and the same for right invariance on $X_{5}\left(\right.$ or $\left.X_{7}\right)$.

Proof. It is sufficient to prove the theorem for $\varphi \in L^{1}(G)$ continuous with compact support. Let $k \in X_{3}$. The map $g \in$ support $\varphi \rightarrow$ $\sigma(g) k\left(g^{-1}\right.$. $) \in X_{3}$ is continuous because, if $g^{\prime} g=g g^{\prime \prime}$,

$$
\begin{aligned}
& \left\|\sigma(g) k\left(g^{-1} \cdot\right)-\sigma\left(g^{\prime} g\right) k\left(\left(g^{\prime} g\right)^{-1} .\right)\right\|_{\infty}=\sup _{v} \mid \sigma(g) k\left(g^{-1} v\right) \\
& \quad-\sigma\left(g g^{\prime \prime}\right) k\left(\left(g g^{\prime \prime}\right)^{-1} v\right)\left|=\sup _{v}\right| k\left(g^{-1} v\right)-\sigma\left(g^{\prime \prime}\right) k\left(g^{\prime \prime-1} g^{-1} v\right) \mid<\varepsilon
\end{aligned}
$$

if $g^{\prime}$ is in some neighborhood of $e$ in $G$. The set $\left\{\sigma(g) k\left(g^{-1}\right), g \in\right.$ support $\varphi\}$ is compact in $X_{3}$ and if

$$
\Phi \in X_{3}^{\prime},\left\langle\Phi, \int \sigma(g) k\left(g^{-1} \cdot\right) \varphi(g) d g\right\rangle=\int\left\langle\Phi, \sigma(g) k\left(g^{-1} \cdot\right)\right\rangle \varphi(g) d g .
$$

Let us choose $\Phi=F \circ M$ with $F \in A^{\prime}$. Then

$$
F\left\{\left\langle M, \int \sigma(g) k\left(g^{-1} .\right) \varphi(g) d g\right\rangle\right\}=\int F\left\{\left\langle M, \sigma(g) k\left(g^{-1} \cdot\right)\right\rangle\right\} \varphi(g) d g .
$$

In the same way, if we choose $\Phi=F \circ \delta_{t}, t \in G$ :

$$
\begin{gathered}
F\left\{\left\langle\delta_{t}, \int \sigma(g) k\left(g^{-1} \cdot\right) \varphi(g) d g\right\rangle\right\}=\int F\left\{\left\langle\delta_{t}, \sigma(g) k\left(g^{-1} \cdot\right)\right\rangle\right\} \varphi(g) d g \\
=\int F\left\{\varphi(g) \sigma(g) k\left(g^{-1} t\right)\right\} d t=F\left\{\left\langle\delta_{t}, \varphi * k\right\rangle\right\} .
\end{gathered}
$$

So $\int \sigma(g) k\left(g^{-1} \cdot\right) \varphi(g) d g=\varnothing * k$ and

$$
\begin{aligned}
& F\{\langle M, \varphi * k\rangle\}=\int F\left\{\varphi(g)\left\langle M, \sigma(g) k\left(g^{-1} \cdot\right)\right\rangle\right\} d g \\
& \quad=\int F\{\varphi(g) \sigma(g)\langle M, k\rangle\} d g=F\left\{\int \varphi(g) \sigma(g)\langle M, k\rangle\right\} d g .
\end{aligned}
$$


This being true for any $F$,

$$
\langle M, \varphi * k\rangle=\int \varphi(g) \sigma(g)\langle M, k\rangle d g .
$$

The proof is similar for left and right invariance.

The next theorem asserts the existence of $\sigma$-left, left and right invariant $A$-means on $G$ if $G$ is amenable.

TheOREM 14. Let $A$ be a Banach-*-algebra such that $A=Z^{\prime}$ (resp. $A$ is a $C^{*}$-algebra). If $G$ is ameanable, there exist $\sigma$-left, left and right invariant means on $G$ with value in $A$ (resp. in $A^{\prime \prime}$ ).

Proof. Let $m$ be an invariant mean on $G, k \in L^{\infty}(G, A), F \in A^{\prime}$ : then $F \circ k \in L^{\infty}(G),\|F \circ k\|_{\infty} \leqq\|F\|\|k\|_{\infty}$ and $\mid\langle m, F \circ k\rangle\|\leqq\| F\|\| k \|_{\infty}$. The correspondence $F \rightarrow\langle m, F \circ k\rangle$ is linear, continuous, and define an element $\langle M, k\rangle$ in the bidual $A^{\prime \prime}$ such that $\langle m, F \circ k\rangle=\langle F\langle M, k\rangle\rangle$ and $|\langle M, k\rangle| \leqq\|k\|_{\infty}$. So the correspondence $k \rightarrow\langle M, k\rangle$ is continuous with norm less than or equal to one. If $k$ and $F$ are positive, $\langle F,\langle M, k\rangle\rangle$ is positive and so $\langle M, k\rangle$ is positive. Moreover, $\left\langle F,\langle M, k a\rangle=\langle m, F \circ k a\rangle=\left\langle m, a^{t} F \circ k\right\rangle=\left\langle a^{t} F,\langle M, k\rangle\right\rangle=\langle F,\langle M, k\rangle a\rangle\right.$ where the product $\langle M, k\rangle a$ is defined through bitransposition [1], [5]. In the same way, $\langle M, a k\rangle=a\langle M, k\rangle$ and $\left\langle F,\left\langle M, \sigma(g) k\left(g^{-1}.\right)\right\rangle\right\rangle=$ $\left\langle m, F \circ \sigma(g) k\left(g^{-1}.\right)\right\rangle=\left\langle m, \sigma(g)^{t} F \circ k\left(g^{-1}.\right)\right\rangle=\left\langle m, \sigma(g)^{t} F \circ k\right\rangle=\left\langle\sigma(g)^{t} F\right.$, $\langle M, k\rangle\rangle=\left\langle F, \sigma(g)^{t t}\langle M, k\rangle\right\rangle$. If $A$ is a $C^{*}$-algebra, the left and right multiplications in $A^{\prime \prime}$ by element from $A$ coincide with the product in the Von Neumann evelopping algebra, while $\sigma(g)^{t t}$ is the natural extension of $\sigma(g)$ to $A^{\prime \prime}$. If $A=Z^{\prime}$, then $\langle M, k\rangle \in A$ as we can see by choosing $F=z \in Z \subset Z^{\prime \prime}=A^{\prime}$ and the left and right multiplication by $a \in A$ is the product in $A$. The same kind of proof shows that $M$ is also left and right invariant.

REMARK. The converse of the preceding theorem is obvious: if, for any Banach-*-algebra $A, G$ is such that there exist invariant $A$-means, then $G$ is ameanable, because it is sufficient to take $A=C$, the complex numbers.

8. The case $n=1$. Bounded derivations and crossed homomorphisms. 1st Part: the "nondual" structure and bounded derivations. Let $T$ be an element of $Z^{1}\left(\mathfrak{A}, \mathfrak{X}_{L}^{*, A}\right)$ : it is a linear and continuous application from $\mathfrak{A}$ to $\mathfrak{X}_{L}^{*}, A$ such that

$$
T\left(f_{1} * f_{2}\right)=T\left(f_{1}\right) * f_{2}+f_{1} * T\left(f_{2}\right) .
$$

By Theorems 8 and 10, its extension $\widetilde{T}$ is also in $Z^{1}\left(\mathfrak{X}_{L}^{*, A}, \mathfrak{X}_{L}^{*, A}\right)$ :

$$
\widetilde{T}\left(\mu_{L} * \nu_{L}\right): \widetilde{T}\left(\mu_{L}\right) * \nu_{L}+\mu_{L} * \widetilde{T}\left(\nu_{L}\right) .
$$


However, in the case $n=1$, it is possible to obtain a more precise result than in Theorem 8:

TheOREM 15. Let $T \in Z^{1}\left(\mathfrak{A}, \mathfrak{X}_{L}^{*, A}\right)$ where $A=Z^{\prime}$ or $A$ is a $C^{*}$ algebra. Then $T$ extends in a unique way as an element $\widetilde{T}$ of $Z^{1}\left(\mathfrak{X}_{L}^{*, A}, \mathfrak{X}_{L}^{*, A}\right)$ which is continuous from $\mathfrak{X}_{L}^{*, A}$ with the strong topology to $\mathfrak{X}_{L}^{*, A}$ with the $\mathfrak{X}$-weak-topology and can be defined by continuous extension:

$$
\left\langle\widetilde{T}\left(\mu_{L}\right), h\right\rangle=\operatorname{limit}_{\alpha, \beta}\left\langle T\left(\mu_{L} * e_{\alpha} \lambda_{\beta}\right), h\right\rangle, \quad h \in \mathfrak{X} .
$$

Moreover, if $T=\Delta \mu_{L}$, then $\widetilde{T}=\Delta \tilde{\mu}_{L}$.

Proof. Our hypothesis on $A$ insure the existence of $\widetilde{T}$ and its uniqueness comes from $\widetilde{T}\left(\mu_{L}\right) * f=T\left(\mu_{L} * f\right)-\mu_{L} * T(f)$, and the last statement of the theorem is evident. Now

$$
\begin{aligned}
& \left\langle T\left(\mu_{L^{\prime}} * e_{\alpha} \lambda_{\beta}\right), h\right\rangle=\left\langle T\left(\mu_{L^{\prime}} * e_{\alpha} \lambda_{\beta}\right) * f, h^{\prime}\right\rangle \\
& \quad=\left\langle T\left(\mu_{L} * e_{\alpha} \lambda_{\beta} * f\right), h^{\prime}\right\rangle-\left\langle\mu_{K} * e_{\alpha} \lambda_{\beta} * T(f), h^{\prime}\right\rangle \\
& =\left\langle T\left(\mu_{L} * e_{\alpha} \lambda_{\beta} * f\right\rangle, h^{\prime}\right\rangle-\left\langle\mu_{L} * e_{\alpha} \lambda_{\beta} * f^{\prime}, h^{\prime \prime}\right\rangle
\end{aligned}
$$

if we write $h=f \cdot h^{\prime}$ and $T(f) \cdot h^{\prime}=f^{\prime} \cdot h^{\prime \prime}$ thanks to the neo-unital character of $\mathfrak{X}$. Taking the limit in the norm of $A$, we have

$$
\begin{aligned}
\operatorname{limit}_{\alpha, \beta} & \left\langle T\left(\mu_{L} * e_{\alpha} \lambda_{\beta}\right), h\right\rangle=\left\langle T\left(\mu_{L} * f\right), h^{\prime}\right\rangle-\left\langle\mu_{L} * f^{\prime}, h^{\prime \prime}\right\rangle \\
& =\left\langle T\left(\mu_{L} * f\right)-\mu_{L} * T(f), h^{\prime}\right\rangle=\left\langle\widetilde{T}\left(\mu_{L}\right) * f, h^{\prime}\right\rangle=\left\langle\widetilde{T}\left(\mu_{L}\right), h\right\rangle
\end{aligned}
$$

which proves the continuity property of $\widetilde{T}$, formula (64) and the fact that $\widetilde{T} \in Z^{1}\left(\mathfrak{X}_{L}^{*}, A, \mathfrak{X}_{L}^{*, A}\right)$.

REMARK. In the case of $Z^{1}\left(\mathfrak{A}, \mathfrak{X}_{L}^{*, A}\right)$, even if $A$ is a $C^{*}$-algebra, $\widetilde{T}$ takes its values in $\mathfrak{X}_{L}^{*}, A$ (and not $\left.\mathfrak{X}^{*, A \prime \prime}\right)$ and $H^{1}\left(\mathfrak{A}, \mathfrak{X}_{L}^{*, A}\right)$ is isomorphic to $H^{1}\left(\mathfrak{X}_{L}^{*}, A, \mathfrak{X}_{L}^{*, A}\right)$ (see Theorem 10). The preceding proof works for $Z^{1}\left(\mathfrak{X}^{*, A}, \mathfrak{X}^{*, A}\right)$ but not for $Z^{1}\left(\mathfrak{X}_{R}^{*, A}, \mathfrak{X}_{R}^{*, A}\right)$. Of course if $T \in Z^{1}\left(\mathfrak{A}, \mathfrak{X}^{*, A \prime \prime}\right)$ then $T \in Z^{1}\left(\mathfrak{X}_{L}^{*, A}, \mathfrak{X}^{*, A^{\prime \prime}}\right)$ and $H^{1}\left(\mathfrak{A}, \mathfrak{X}^{*, A^{\prime \prime}}\right)$ is isomorphic to $H^{1}\left(\mathfrak{X}_{L}^{*, A}, \mathfrak{X}^{*, A^{\prime \prime}}\right)$.

Let us now consider the following function, linear on $A$,

$$
(g, a) \in G \times A \longrightarrow\left\langle\widetilde{T}\left(a \delta_{g}\right), h\right\rangle \in A, T \in Z^{1}\left(\mathfrak{A}, \mathfrak{X}_{L}^{*}, A\right) .
$$

Because $\mid\left\langle\widetilde{T}\left(a \delta_{g}, h\right\rangle\right|\left\langle\|T\||a|\left\langle h \|_{\infty}\right.\right.$, we can write $\left\langle\widetilde{T}\left(a \delta_{g}\right), h\right\rangle=$ $\left\langle V_{T}(g) a, h\right\rangle$ where $V_{T}(g) \in \mathscr{L}\left(A, \mathfrak{X}_{L}^{*, A}\right)$ and $\left\langle\left\langle V_{T}(g) a, h\right\rangle \in L^{\infty}(G, A)\right.$ : we recover the function $V_{T}$ of Theorems 6 and 9 . Of course,

$$
\left\{\begin{array}{l}
\widetilde{T}\left(a \delta_{g} * b \delta_{g^{\prime}}\right)=\widetilde{T}\left(a \delta_{g}\right) * b \delta_{g^{\prime}}+a \delta_{g} * \widetilde{T}\left(b \delta_{g^{\prime}}\right) \\
V_{T}\left(g g^{\prime}, a \sigma(g) b\right)=V_{T}(g) a * b \delta_{g^{\prime}}+a \delta_{g} * V_{T}\left(g^{\prime}\right) b .
\end{array}\right.
$$

Conversely, let 


$$
(g, a) \in G \times A \longrightarrow F(g, a) \in \mathfrak{X}_{L}^{*, A}
$$

a mapping, linear on $A$, such that $\langle F(g, a), h\rangle \in L^{\infty}(G, A),\|F\|=$ $\sup _{|a| \leq 1, g \in G}\|F(g, a)\|_{1}<\infty$ and $F\left(g g^{\prime}, a \sigma(g) b\right)=F(g, a) * b \delta_{g^{\prime}}+a \delta_{g} * F\left(g^{\prime}, b\right)$. Then there exists $V(g) \in \mathscr{L}\left(A, \mathfrak{X}_{L}^{*, A}\right)$ such that $\langle F(g, a), h\rangle=\langle V(g) a, h\rangle$ and $F$ extends from $G \times A$ to $\mathfrak{A}$ by

$$
\langle F(a \varphi), h\rangle=\int \varphi(g)\langle F(g, a), h\rangle d g, \quad \varphi \in L^{1}(G)
$$

and continuous extension. Then $F \in Z^{1}\left(\mathfrak{A}, \mathfrak{X}_{L}^{*, A}\right)$ because

$$
\begin{aligned}
\left\langle F\left(a \varphi_{1}\right) * b \varphi_{2}, h\right\rangle=\left\langle F\left(a \varphi_{1}\right)(u),\left\langle\sigma(u) b \varphi_{2}(v), h(u v)\right\rangle\right\rangle \\
\quad=\int \varphi_{1}(g)\left\langle F(g, a)(u),\left\langle\sigma(u) b \varphi_{2}(v), h(u v)\right\rangle d g\right. \\
\quad=\int \varphi_{1}(g)\left\langle F(g, a)(u), \int \varphi_{2}(w)\left\langle\sigma(u) b \delta_{w}(v), h(u v)\right\rangle d w\right\rangle d g \\
=\int \varphi_{1}(g) \int \varphi_{2}(w)\left\langle F(g, a)(u),\left\langle\sigma(u) b \delta_{w}(v), h(u v)\right\rangle d w d g\right. \\
=\int \varphi_{1}(g) \varphi_{2}(w)\left\langle F(g, a) * b \delta_{w}, h\right\rangle d w d g
\end{aligned}
$$

where we used Fubini's theorem to interchange the measures defined by $F(g, a)$ and $\varphi_{2}$, while on the other hand,

$$
\begin{aligned}
\left\langle a \varphi_{1} *\right. & \left.F\left(b \varphi_{2}\right), h\right\rangle=\left\langle a \varphi_{1}(u),\left\langle{ }^{u} F\left(b \varphi_{2}\right)(v), h(u v)\right\rangle\right\rangle \\
& =a \int \varphi_{1}(u) \sigma(u)\left\langle F\left(b \varphi_{2}\right)(v), \sigma\left(u^{-1}\right) h(u v)\right\rangle d u \\
& =a \int \varphi_{1}(u) \sigma(u) \int \varphi_{2}(w)\left\langle F(b, w)(v), \sigma\left(u^{-1}\right) h(u v)\right\rangle d w d u \\
& =a \int \varphi_{1}(u) \int \varphi_{2}(w)\left\langle{ }^{u} F(b, w)(v), h(u v)\right\rangle d w d u \\
& =\int \varphi_{2}(w) \int a \varphi_{1}(u)\left\langle{ }^{u} F(b, w)(v), h(u v)\right\rangle d u d w \\
& =\int \varphi_{2}(w) \varphi_{1}(g)\left\langle a \delta_{g}(u),\left\langle{ }^{u} F(b, w)(v), h(u v)\right\rangle d g d w\right. \\
& =\int \varphi_{1}(g) \varphi_{2}(w)\left\langle a \delta_{g} * F(b, w), h\right\rangle d g d w
\end{aligned}
$$

thanks, once again, to Fubini's theorem which is valid if we take for instance $\varphi_{1}$ and $\varphi_{2}$ with compact support. So, if we add the two preceding results,

$$
\begin{aligned}
& \left\langle F\left(a \varphi_{1}\right) * b \varphi_{2}+a \varphi_{1} * F\left(b \varphi_{2}\right), h\right\rangle \\
& \quad=\int \varphi_{1}(g) \varphi_{2}(w)\left\langle F(g, a) * b \delta_{w}+a \delta_{g} * F(b, w), h\right\rangle d w d g
\end{aligned}
$$




$$
\begin{aligned}
& =\int \varphi_{1}(g) \varphi_{2}(w)\langle F(g w, a \sigma(g) b), h\rangle d w d g \\
& =\int \varphi_{1}(g) \varphi_{2}\left(g^{-1} w^{\prime}\right)\left\langle F\left(w^{\prime}, a \sigma(g) b, h\right\rangle d w^{\prime} d g\right. \\
& =\left\langle F\left(\int \varphi_{1}(g) \varphi_{2}\left(g^{-1} w^{\prime}\right) a \sigma(g) b d g\right), h\right\rangle \\
& =\left\langle F\left(a \varphi_{1} * b \varphi_{2}\right), h\right\rangle .
\end{aligned}
$$

Of course the preceding discussion can be adapted to $T \in Z^{1}\left(\mathfrak{A}, \mathfrak{X}^{*, A^{\prime \prime}}\right)$.

Let us now consider the three functions on $G$ with value in $\mathfrak{X}_{L}^{*, A}$ :

$$
\left\{\begin{array}{l}
\chi(g)=\widetilde{T}\left(\delta_{g}\right) * \delta_{g^{-1}}=-\delta_{g} * \widetilde{T}\left(\delta_{g}-1\right) \\
\chi^{a}(g)=\widetilde{T}\left(a \delta_{g}\right) * \delta_{g^{-1}}=\widetilde{T}\left(a \delta_{0}\right)-a \delta_{g} * \widetilde{T}\left(\delta_{g^{-1}}\right), a \in A \\
a^{\prime}(g)=\delta_{g} * \widetilde{T}\left(\sigma \left(g^{-1}\left(a \delta_{0}\right) * \delta_{g^{-1}}, a \in A\right.\right.
\end{array}\right.
$$

for $T \in Z^{1}\left(\mathfrak{A}, \mathfrak{X}_{L}^{*, A}\right)$.

Lemma 2. For any $h \in \mathfrak{A}$ and $a \in A$, the three functions $\langle\chi(g), h\rangle$, $\left\langle\chi^{a}(g), h\right\rangle$ and $\left\langle{ }^{a} \chi(g), h\right\rangle$ are in the space $X_{2}$ (see paragraph 7 ).

Proof. These three functions are bounded by $\|T\|\|h\|_{\infty}$ and $\|T\|\|h\|_{\infty}|a|$ respectively. Moreover,

$$
\begin{aligned}
& \left\langle\chi^{a}(g)-\chi^{a}\left(g^{\prime}\right), h\right\rangle \leqq\left|\left\langle\widetilde{T}\left(a \delta_{g}\right)-\widetilde{T}\left(a \delta_{g^{\prime}}\right), g^{-1} \cdot h\right\rangle\right| \\
& +\left|\left\langle\widetilde{T}\left(a \delta_{g^{\prime}}\right), g^{-1} \cdot h-g^{-1} \cdot h\right\rangle\right| \\
& \leqq\left|\left\langle\widetilde{T}\left(a \delta_{g}\right)-\widetilde{T}\left(a \delta_{g^{\prime}}\right), g^{-1} \cdot h\right\rangle\right|+\|T\||a|\left\|g^{-1} \cdot h-g^{\prime-1} \cdot h\right\|_{\infty} \\
& \leqq 2 \varepsilon+\left|\left\langle T\left(a \delta_{g} * e_{\alpha} \lambda_{\beta}\right)-T\left(a \delta_{g^{\prime}} * e_{\alpha} \lambda_{\beta}\right)\right\rangle\right|+\|T\||a|\left\|g^{-1} \cdot h-g^{\prime-1} \cdot h\right\|_{\infty}
\end{aligned}
$$

for $\alpha$ and $\beta$ large enough, and less than $4 \varepsilon$ for $g^{\prime}$ in a suitable neighborhood of $g$. The proof for $\chi(g)$ and ${ }^{a} \chi(g)$ would be similar.

Proposition 10. Let us assume there exists a $\sigma$-left invariant $A$ (resp. $A^{\prime \prime}$ )-mean on $X_{2}$ (for instance $G$ ameanable). The formulas

$$
\left\{\begin{array}{l}
\left\langle\mu_{L}, h\right\rangle=M\{\langle\chi(g), h\rangle\} \\
\left\langle\mu_{L}^{a}, h\right\rangle=M\left\{\left\langle\chi^{a}(g), h\right\rangle\right\} \\
\left\langle{ }^{a} \mu_{L}, h\right\rangle=M\left\{\left\langle{ }^{a} \chi(g), h\right\rangle\right\}
\end{array}\right.
$$

define $\mu_{L}, \mu_{L}^{a}$ and ${ }^{a} \mu_{L}$ as elements of $\mathfrak{X}_{L}^{*, A}$ (resp. $\mathfrak{X}^{\left.*, A^{\prime \prime}\right)}$ related by

$$
\left\{\begin{array}{l}
\mu_{L}^{x}=\widetilde{T}\left(a \delta_{0}\right)+a \delta_{0} * \mu_{L}=\mu_{L} * a \delta_{0}+{ }^{a} \mu_{L} \\
{ }^{a} \mu_{L}=\widetilde{T}\left(a \delta_{0}\right)+a \delta_{0} * \mu_{L}-\mu_{L} * a \delta_{0}=\widetilde{T}\left(a \delta_{0}\right)+\widetilde{\Delta \tilde{\mu}_{L}}\left(a \delta_{0}\right) .
\end{array}\right.
$$

Proof. The right hand sides of formulas (70) have a meaning 
by Lemma 2. They are $A$-right linear in $h$ and if $\left(h_{i}\right)$ is a finite family in $K(G, A)$ with support $h_{i} \cap$ support $h_{j}=\varnothing$ for $i \neq j$ and $\left\|\sum_{i} h_{i}\right\|_{\infty} \leqq 1$, then

$$
\sum_{i}\left|M\left\{\left\langle\chi(g), h_{i}\right\rangle\right\}\right| \leqq \sum_{i} \sup _{g}\left|\left\langle\chi(g), h_{i}\right\rangle\right| \leqq \sup _{g}\|\chi(g)\|_{1} \leqq\|T\|
$$

which proves that $\mu_{L} \in \mathfrak{X}_{L}^{*, 4}$ (resp. $\mathfrak{X}^{*, L^{\prime \prime}}$ ) with $\left\|\mu_{L}\right\|_{1} \leqq\|T\|$. In the same way $\mu_{L}^{2}$ and ${ }^{a} \mu_{L} \in \mathfrak{X}_{L}^{*, A}$ (resp. $\mathfrak{X}^{*, A^{\prime \prime}}$ ) with norm less than $\|T\||a|$. Formulas (71) comes from the equality between

$$
\widetilde{T}\left(a \delta_{g}\right) * \delta_{g^{-1}}=\widetilde{T}\left(a \delta_{0} * \delta_{g}\right) * \delta_{g^{-1}}=\widetilde{T}\left(a \delta_{0}\right)+a \delta_{0} * \widetilde{T}\left(\delta_{g}\right) * \delta_{g^{-1}}
$$

and

$$
\begin{aligned}
\widetilde{T}\left(a \delta_{g}\right) * \delta_{g-1} & =\widetilde{T}\left(\delta_{g} * \delta_{g-1} * a \delta_{0} * \delta_{g}\right) * \delta_{g-1} \\
& =\widetilde{T}\left(\delta_{g}\right) * \delta_{g-1} * a \delta_{0}+\delta_{g} * \widetilde{T}\left(\sigma\left(g^{-1}\right) a \delta_{0}\right) * \delta_{g-1}
\end{aligned}
$$

THeOREM 16. Let $T \in Z^{1}\left(\mathfrak{A}, \mathfrak{X}_{L}^{*, A}\right)$ (or $Z^{1}\left(\mathfrak{A}, \mathfrak{X}^{*, 4^{\prime \prime}}\right)$ ) and $M$ a $\sigma$-left invariant $A$ (or $A^{\prime \prime}$ )-mean or $X_{2}$ (this is the case if $G$ is ameanable). Then

$$
\left\{\begin{array}{l}
\widetilde{T}\left(a \delta_{g}\right)=\widetilde{T}\left(a \delta_{0}\right) * \delta_{g}-a \delta_{0} * \Delta \widetilde{\mu}_{L}\left(\delta_{g}\right)=-\widetilde{\Delta \mu_{L}}\left(a \delta_{g}\right)+{ }^{a} \mu_{L} * \delta_{g} \\
\widetilde{T}\left(\delta_{g}\right)=-\widetilde{\Delta \mu_{L}}\left(\delta_{g}\right) \\
\widetilde{T}\left(a \delta_{0}\right)=-\widetilde{\Delta \mu_{L}}(a \delta)+{ }^{a} \mu_{L}
\end{array}\right.
$$

and

$$
\left\{\begin{array}{l}
T(a \varphi)=\widetilde{T}\left(a \delta_{0}\right) * \varphi-a \delta_{0} * \widetilde{\Delta \mu}_{L}(\varphi)=-\Delta \mu_{L}(a \varphi)+{ }^{a} \mu_{L} * \varphi \\
\widetilde{T}(\varphi)=-\Delta \widetilde{\mu}_{L}(\varphi) .
\end{array}\right.
$$

Moreover,

$$
\sigma(g) a \widetilde{\Delta \mu_{L}}\left(\delta_{g}\right)-\widetilde{T}\left(\sigma(g) a \delta_{0}\right) * \delta_{g}=\widetilde{\Delta \mu_{L}}\left(\delta_{g}\right) * a \delta_{0}-\delta_{g} * \widetilde{T}\left(a \delta_{0}\right)
$$

or, equivalently

$$
\sigma(g)^{a} \mu_{L} * \delta_{g}=\delta_{g} *^{a} \mu_{L}
$$

If $\widetilde{T}$ restricted to $A$ is inner, (i.e., is a coboundary), then $T$ is inner.

Conversely, if $t \in Z^{1}\left(A, \mathfrak{X}_{L}^{*, A}\right)$ (or $Z^{1}\left(A, \mathfrak{X}^{*, A^{\prime \prime}}\right)$ ) and $\mu_{L} \in \mathfrak{X}_{L}^{*, A}$ (or $\left.\mathfrak{X}^{*, A}\right)$ are related according to $(74)$ or $(75)$ where ${ }^{a} \mu_{L}$ is defined by (71), then formulas (72) and (73) define an element $T$ of $Z^{1}\left(\mathfrak{A}, \mathfrak{X}_{L}^{*, A}\right)$ (or $\left.Z^{1}\left(\mathfrak{A}, \mathfrak{X}^{*, A^{\prime \prime}}\right)\right)$. If $t$ is inner then $T$ is inner.

Finally, if $T(\mathfrak{A}) \subset \mathfrak{A}$, i.e., if $T$ is a derivation on $\mathfrak{A}$, then $\widetilde{T}(A) \subset$ $M_{1}(G, A)$ and $\mu_{L} \in \mathfrak{X}^{*, A} \cap M_{1}(G, M(A))$, and conversely. 
Proof.

$$
\begin{aligned}
& \left\langle a \delta_{g} * \mu_{L}, h\right\rangle=a\left\langle\delta_{g} * \mu_{L}, h\right\rangle=a\left\langle{ }^{g} \mu_{L}, h . g\right\rangle \\
& \quad=a \sigma(g)\left\langle\mu_{L}, \sigma\left(g^{-1}\right) h . g\right\rangle=a \sigma(g) M\left\{\left\langle\chi(u), \sigma\left(g^{-1}\right) h . g\right\rangle\right\} \\
& \quad=a M\left\{\sigma(g)\left\langle\chi\left(g^{-1} u\right), \sigma\left(g^{-1}\right) h . g\right\rangle\right\} \\
& \quad=M\left\{a\left\langle{ }^{g} \chi\left(g^{-1} u\right), h . g\right\rangle\right\}=M\left\{a\left\langle g . \chi\left(g^{-1} u\right), h\right\rangle\right\} \\
& \quad=M\left\{\left\langle a \delta_{g} * \widetilde{T}\left(\delta_{g^{-1}}\right) * \delta_{u}{ }^{-1}, h\right\rangle\right\} \\
& \quad=M\left\{\left\langle\widetilde{T}\left(a \delta_{u}\right) * \delta_{u^{-1}}, g . h\right\rangle\right\}-M\left\{\left\langle\widetilde{T}\left(a \delta_{g}\right), h\right\rangle\right\} \\
& \quad=\left\langle\mu_{L}^{x}, g . h\right\rangle-\left\langle\widetilde{T}\left(a \delta_{g}\right), h\right\rangle .
\end{aligned}
$$

So $\widetilde{T}\left(a \delta_{g}\right)=\mu_{L}^{2} * \delta_{g}-a \delta_{g} * \mu_{L}$ which gives (72) with the help of (71). Formulas (73) are then coming from $\langle T(\alpha \varphi), h\rangle=\int \varphi(g)\left\langle\widetilde{T}\left(a \delta_{g}\right), h\right\rangle d g$. We obtain formula (74) from the equality between

$$
\widetilde{T}\left(\sigma(g) a \delta_{g}\right)=\widetilde{T}\left(\sigma(g) a \delta_{0} * \delta_{g}\right)=\widetilde{T}\left(\sigma(g) a \delta_{0}\right) * \delta_{g}+\sigma(g) a \delta_{0} * \widetilde{T}\left(\delta_{g}\right)
$$

and

$$
\widetilde{T}\left(\sigma(g) a \delta_{g}\right)=\widetilde{T}\left(\delta_{g} * a \delta_{0}\right)=\delta_{g} * \widetilde{T}\left(a \delta_{0}\right)+\widetilde{T}\left(\delta_{0}\right) * a \delta_{0}
$$

equality which gives also (75) with the help of (71).

If $\widetilde{T}\left(a \delta_{0}\right)=-\widetilde{\Delta \mu^{\prime}}\left(a \delta_{0}\right)$, then $\widetilde{T}+\widetilde{\Delta \mu^{\prime}}$ is zero on $A$ and the corresponding ${ }^{a} \mu_{L}^{\prime}$ is zero, so $\widetilde{T}+\widetilde{\Delta \mu^{\prime}}=-\widetilde{\Delta \mu_{L}^{\prime}}$ or $T=-\Delta\left(\mu^{\prime}+\mu_{L}^{\prime}\right)$.

Conversely, given $t$ and $\mu_{L}$, let $\widetilde{T}\left(a \delta_{g}\right)=t\left(a \delta_{0}\right) * \delta_{g}-a \delta_{0} * \widetilde{\Delta \mu_{L}}\left(\delta_{g}\right)$. Then

$$
\begin{aligned}
& \widetilde{T}\left(a \delta_{g} * b \delta_{g^{\prime}}\right)=\widetilde{T}\left(a \sigma(g) b \delta_{g g^{\prime}}\right) \\
& =t\left(a \sigma(g) b \delta_{0}\right) * \delta_{g g^{\prime}}-a \sigma(g) b \delta_{0} * \widetilde{\Delta} \mu_{L}\left(\delta_{g g^{\prime}}\right) \\
& =t\left(a \delta_{0} * \sigma(g) b \delta_{0}\right) * \delta_{g g^{\prime}}-a \delta_{g} * b \delta_{g^{-1}} * \widetilde{\Delta \mu_{L}}\left(\delta_{g g^{\prime}}\right) \\
& =t\left(a \delta_{0}\right) * \sigma(g) b \delta_{g g^{\prime}}+a \delta_{0} * t\left(\sigma(g) b \delta_{0}\right) * \delta_{g g^{\prime}}-a \delta_{g} * b \delta_{g}-1^{\prime} * \widetilde{\Delta \mu}_{L}\left(\delta_{g g^{\prime}}\right) \\
& =t\left(a \delta_{0}\right) * \delta_{g} * b \delta_{g^{\prime}}+a \delta_{g} * \delta_{g}-1 * t\left(\sigma(g) b \delta_{0}\right) * \delta_{g g^{\prime}}-a \delta_{g} * b \delta_{g}-1 * \widetilde{\Delta} \mu_{L}\left(\delta_{g}\right) * \delta_{g^{\prime}} \\
& -a \delta_{g} * b \delta_{g-1} * \delta_{g} * \widetilde{\Delta \mu}_{L}\left(\delta_{g^{\prime}}\right) \\
& =t\left(a \delta_{0}\right) * \delta_{g} * b \delta_{g^{\prime}}-a \delta_{g} * b \delta_{g}-1 * \widetilde{\Delta \mu} \tilde{\mu}_{L}\left(\delta_{g}\right) * \delta_{g} \text {, }
\end{aligned}
$$

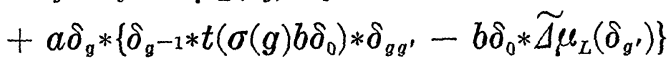

$$
\begin{aligned}
& =\left\{t\left(a \delta_{0}\right) * \delta_{g} * b \delta_{g^{\prime}}-a \sigma(g) b \delta_{0} * \widetilde{\Delta \mu_{L}}\left(\delta_{g}\right) * \delta_{g^{\prime}}\right. \\
& +a \delta_{g} *\left\{\delta_{g-1} * t\left(\sigma(g) b \delta_{0}\right) * \delta_{g g^{\prime}}-b \delta_{0} * \widetilde{\Delta \mu_{L}}\left(\delta_{g^{\prime}}\right)\right\}
\end{aligned}
$$

which, thanks to (74), gives

$$
\begin{gathered}
t\left(a \delta_{0}\right) * \delta_{g} * b \delta_{g^{\prime}}-a \delta_{0} * \widetilde{\Delta \mu_{L}}\left(\delta_{g}\right) * b \delta_{g^{\prime}}+a \delta_{g^{\prime}} *\left\{t\left(b \delta_{0}\right) * \delta_{g^{\prime}}-b \delta_{0} * \widetilde{\Delta \mu_{L}}\left(g^{\prime}\right)\right\} \\
=\widetilde{T}\left(a \delta_{g}\right) * b \delta_{g^{\prime}}+a \delta_{g} * \widetilde{T}\left(b \delta_{g^{\prime}}\right) .
\end{gathered}
$$

Finally, if $T(a \varphi) \in \mathfrak{A}$ for any $a, \varphi$, this is equivalent to $\widetilde{T}\left(a \delta_{0}\right) * \varphi$, $a \delta_{0} * \mu_{L} * \varphi$ and $a \varphi * \mu_{L}$ be in $\mathfrak{U}$ for any $a$ and $\varphi$. The last condition 
means that $\mu_{L} \in \mathfrak{X}^{*, A}$ instead of $\mathfrak{X}_{L}^{*, A}$. If $\mu_{L} \in \mathfrak{X}_{L}^{*, A}$ and $\varphi \in L^{1}(G)$, it is easy to see that $\mu_{L} * \varphi \in L^{1}\left(G, M_{L}(A)\right)$. If $\varphi=\xi_{B}$, the characteristic function of some Borel $B \in B(G)$, the first condition reads $\left\{\widetilde{T}\left(a \delta_{0}\right) *\right.$ $\varphi\}(g)=\widetilde{T}\left(a \delta_{0}\right)\left(g B^{-1}\right) \in A$ or else $\widetilde{T}\left(a \delta_{0}\right) \in M_{1}(G, A)$. The second condition reads $a \mu_{L}\left(g^{-1} B\right) \in A$, which means that $\mu_{L} \in M_{1}(G, M(A))$.

If $G$ is discrete, this result is very close to ([17, Theorem 1]).

Definition. $T \in Z^{1}\left(\mathfrak{A}, \mathfrak{X}_{L}^{*}, A\right)$, will be called special if $\widetilde{T}(A) \subset A$, i.e., $\widetilde{T}\left(a \delta_{0}\right)=t(a) \delta_{0}$ where $t$ is a derivation on $A$.

THEOREM 17. Let $T \in Z^{1}\left(\mathfrak{A}, \mathfrak{X}_{L}^{*, A}\right)$ be special. Then $T$ is equivalent to the couple $\left(t, \mu_{L}\right)$ where $t$ is a derivation on $A$ and $\mu_{L}$ an element of $\mathfrak{X}_{L}^{*, A}$ related by $(74)$ or $(75)$ (where $\left.\widetilde{T}\left(a \delta_{0}\right)=t(a) \delta_{0}\right)$. If $t$ is inner, then $T$ is inner. Finally $T(\mathfrak{U}) \subset \mathfrak{A}$, i.e., $T$ is a special derivation on $\mathfrak{A}$, if $\mu_{L} \in \mathfrak{X}^{*, A} \cap M_{1}(G, M(A))$ and conversely. If $T \in Z^{1}\left(\mathfrak{A}, \mathfrak{X}^{*, A^{\prime \prime}}\right)$ is special, $\mu_{L} \in \mathfrak{X}^{*, A^{\prime \prime}}$ and conversely.

Proof. It is an adaptation of Theorem 16, noticing that condition $\widetilde{T}\left(a \delta_{0}\right) \in M_{1}(G, A)$ is now automatic.

THEOREM 18. If $T$ is such that $\delta_{g} * \widetilde{T}\left(\sigma\left(g^{-1}\right) a \delta_{0}\right) * \delta_{g-1}=\widetilde{T}\left(a \delta_{0}\right)$ (which, in the case of a special $T$ means that $t(\sigma(g) a)=(\sigma(g) t(a))$, then ${ }^{a} \mu_{L}=\widetilde{T}\left(a \delta_{0}\right)$ or, equivalently, $a \delta_{0} * \mu_{L}=\mu_{L} * a \delta_{0}$ (i.e., $\widetilde{\Delta \mu_{L}}\left(a \delta_{0}\right)=0$ ), and conversely.

Proof. It is a straightforward application of Theorem 16.

2nd part: The "dual" structure and crossed homomorphisms. All what has been done in the first part can be adapted to the "dual" structure. An element $T$ of $Z^{1}\left(\mathfrak{A}, \mathfrak{X}_{L}^{*}, A\right)$ is now an affine and continuous application from $\mathfrak{A}$ to $\mathfrak{X}_{L}^{*, A}$ such that

$$
T\left(f_{1} * f_{2}\right)=T\left(f_{1}\right) * f_{2}+T\left(f_{2}\right) .
$$

Theorem 15 works in exactly the same way, proving the uniqueness of $\widetilde{T} \in Z^{1}\left(\mathfrak{X}_{L}^{*}, A, \mathfrak{X}_{L}^{*, A}\right)$ with

$$
\widetilde{T}\left(\mu_{L} * \nu_{L}\right)=\widetilde{T}\left(\mu_{L}\right) * \nu_{L}+\widetilde{T}\left(\nu_{L}\right) .
$$

Substituting to the affine application $T$ the linear one $T_{1}=T-$ $T(0)$, we can repeat all what has been done in the preceding part, the only change being a new definition of $a \chi(g)$ :

$$
{ }^{a} \chi(g)=\widetilde{T}\left(\sigma\left(g^{-1}\right) a \delta_{0}\right) * \delta_{g}-1
$$

and the use of a left invariant mean instead of $\sigma$-left invariant one, 
so that now

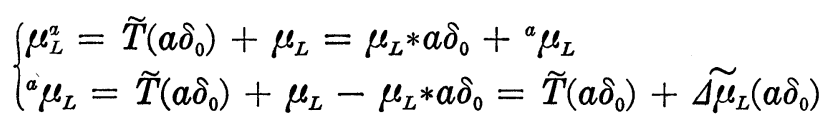

and we obtain the equivalent of Theorem 16:

THEOREM $16^{\text {bis }}$. Let $T \in Z^{1}\left(\mathfrak{A}, \mathfrak{X}_{L}^{*, A}\right)\left(\right.$ or $\left.Z^{1}\left(\mathfrak{A}, \mathfrak{X}^{*, A^{\prime \prime}}\right)\right)$ and $M a$ left invariant $A$ (or $\left.A^{\prime \prime}\right)$-mean on $X_{2}$ (this is the case if $G$ is ameanable). Then

$$
\left\{\begin{array}{l}
\widetilde{T}\left(a \delta_{g}\right)=\widetilde{T}\left(a \delta_{0}\right) * \delta_{g}-\widetilde{\Delta \mu_{L}}\left(\delta_{g}\right)=-\widetilde{\Delta \mu_{L}}\left(a \delta_{g}\right)+{ }^{a} \mu_{L} * \delta_{g} \\
\widetilde{T}\left(\delta_{g}\right)=-\widetilde{\Delta \mu_{L}}\left(\delta_{g}\right) \\
\widetilde{T}\left(a \delta_{0}\right)=-\widetilde{\Delta \mu_{L}}\left(a \delta_{0}\right)+{ }^{a} \mu_{L}
\end{array}\right.
$$

and

$$
\left\{\begin{array}{l}
T(a \varphi)=\widetilde{T}\left(a \delta_{0}\right) * \varphi-{\widetilde{\Delta \mu_{L}}}_{L}(\varphi)=-\Delta \mu_{L}(a \varphi)+{ }^{a} \mu_{L} * \varphi \\
\widetilde{T}(\varphi)=-\Delta \mu_{L}(\varphi) .
\end{array}\right.
$$

Moreover

$$
\widetilde{\Delta \mu_{L}}\left(\delta_{g}\right)-T\left(\sigma(g) a \delta_{0}\right) * \delta_{g}=\widetilde{\Delta \mu_{L}}\left(\delta_{g}\right) * a \delta_{0}-\widetilde{T}\left(a \delta_{0}\right)
$$

or, equivalently,

$$
{ }^{\sigma(g) a} \mu_{L} * \delta_{g}={ }^{a} \mu_{L}
$$

If $\widetilde{T}$ restricted to $A$ is inner (i.e., is a coboundary), then $T$ is inner.

Conversely, if $t \in Z^{1}\left(A, \mathfrak{X}_{L}^{*, A}\right)$ (or $Z^{1}\left(A, \mathfrak{X}^{*, A^{\prime \prime}}\right)$ ) and $\mu_{L} \in \mathfrak{X}_{L}^{*, A}$ (or $\left.\mathfrak{X}^{*, A^{\prime \prime}}\right)$ are related according to (82) or (83), then formulas (80) and (81) define an element $T \in Z^{1}\left(\mathfrak{A}, \mathfrak{X}_{L}^{*, A}\right)$ (or $Z^{1}\left(\mathfrak{A}, \mathfrak{X}^{*, A^{\prime \prime}}\right)$ ). If $t$ is inner then $T$ is inner.

Finally, if $T(A) \subset \mathfrak{A}$, i.e., if $T$ is crossed homomorphism on $\mathfrak{A}$, $\widetilde{T}(A) \subset M_{1}(G, A)$ and $\mu_{L} \in \mathfrak{A}$, and conversely.

Definition. $\mathrm{T} \in Z^{1}\left(\mathfrak{A}, \mathfrak{X}_{L}^{*}, A\right)$ will be called special if $\widetilde{T}(A) \subset A$, i.e., $\widetilde{T}\left(a \delta_{0}\right)=t(a) \delta_{0}$ where $t$ is a crossed homomorphism on $A$.

TheOREM $17^{\text {bis }}$. Let $T \in Z^{1}\left(\mathfrak{A}, \mathfrak{X}_{L}^{*, A}\right)$ be special. Then $T$ is equivalent to the couple $\left(t, \mu_{L}\right)$ where $t$ is a crossed homomorphism on $A$ and $\mu_{L}$ an element of $\mathfrak{X}_{L}^{*, A}$ related by (82) or (83). If $t$ is inner, then $T$ is inner. Finally $T(\mathfrak{U}) \subset \mathfrak{A}$ i.e., $T$ is a special crossed homomorphism on $\mathfrak{A}$ if $\mu_{L} \in \mathfrak{A}$ and conversely. If $T \in Z^{1}\left(\mathfrak{A X},{ }^{*}{ }^{\prime \prime}\right)$ is special, $\mu_{L} \in \mathfrak{X}^{*, A^{\prime \prime}}$ and conversely. 
The equivalent of Theorem 18 gives now rather trivial results.

THEOREM $18^{\text {bis }}$. If $T$ is such that $\widetilde{T}\left(\sigma\left(g^{-1}\right) a \delta_{0}\right) * \delta_{g^{-1}}=\widetilde{T}\left(a \delta_{0}\right)$ (which in the case of a special crossed homomorphism means that $t(\sigma(g) a) \delta_{g}=t(a) \delta_{0}$, i.e., $\left.t=0\right)$ then ${ }^{a} \mu_{L}=\widetilde{T}\left(a \delta_{0}\right)$ or, equivalently, $\mu_{L}=$ $\mu_{L} * a \delta_{0}$ for any $a$, i.e., $\mu_{L}=0$, and conversely.

Acknowledgments. The author achieved this work as he was a visiting professor at The Pennsylvania State University and would like to take this opportunity for expressing his grateful acknowledgments to Professor D. C. Rung. He is also grateful to Professor Bunce for pointing out reference [17].

\section{REFERENCES}

1. R.F. Arens, The adjoint of a bilinear operation, Proc. Amer. Math. Soc., 2 (1951), 839-848.

2. R. C. Busby, Centralizers of twisted group algebras, Pacific J. Math., 47 (1973), 357-392.

3. - Double centralizers and extensions of $C^{*}$-algebras, Trans. Amer. Math. Soc., 132 (1968), 79-99.

4. R. C. Busby and H.A. Smith, Representations of twisted group algebras, Trans. Amer. Math. Soc., 149 (1970), 503-537.

5. P. Civin and B. Yood, The second conjugate space of a Banach algebra as an algebra, Pacific J. Math., 11 (1961), 847-870.

6. P.C. Curtis and A. Figa-Talamanca, Function Algebras, Scott, Foresman and Co., Chicago, 1966.

7. N. Dinculeanu, Vector Measures, Pergamon Press, New York, 1967.

8. S. Doplicher, D. Kastler and D. Robinson, Covariance algebras in field theory and statistical mechanics, Commun. Math. Phys., 3 (1966), 1-28.

9. S. Doplicher, R. V. Kadison, D. Kastler and D. W. Robinson, Asymptotically abelian systems, Commun. Math. Phys., 6 (1967), 101-120.

10. F. P. Greenleaf, Invariant Means on Topological Groups, Van Nostrand, New York, 1957.

11. A. Grothendieck, Produits tensoriels topologiques et espaces nucléaires, Memoirs Amer. Math. Soc., 16 (1955).

12. E. Hewitt and K. A. Ross, Abstract Harmonic Analysis, Vol. II, Springer, Berlin, 1970 .

13. - Abstract Harmonic Analysis, Vol. I, Springer, Berlin, 1963.

14. G. Hochschild, On the cohomology groups of an associative algebra, Ann. of Math., 46 (1945), 58-67.

15. B. E. Johnson, Cohomology in Banach Algebras, Memoirs Amer. Math. Soc., 127 (1972).

16. - An introduction to the theory of centralizers, Proc. London Math. Soc., 14 (1964), 299-320.

17. J. Rosenberg, Amenability of cross products of $C^{*}$-algebras, Preprint, University of Pennsylvania.

Received February 28, 1978.

Université des Sciences et Techniques du Languedoc 34060 Montpellier, France 


\section{PACIFIC JOURNAL OF MATHEMATICS}

\section{EDITORS}

DONALD BABBITT (Managing Editor)

University of California

Los Angeles, CA 90024

HUGO RossI

University of Utah

Salt Lake City, UT 84112

C. C. Moore and ANDrew OGG

University of California

Berkeley, CA 94720
J. DUGUNDJI

Department of Mathematics University of Southern California Los Angeles, CA 90007

R. FINN and J. MILGRAM

Stanford University

Stanford, CA 94305

\section{ASSOCIATE EDITORS}
E. F. BeCKENBACH
B. H. NeumanN
F. WOLF
K. YOSHIDA

\section{SUPPORTING INSTITUTIONS}

UNIVERSITY OF BRITISH COLUMBIA CALIFORNIA INSTITUTE OF TECHNOLOGY UNIVERSITY OF CALIFORNIA MONTANA STATE UNIVERSITY UNIVERSITY OF NEVADA, RENO NEW MEXICO STATE UNIVERSITY OREGON STATE UNIVERSITY UNIVERSITY OF OREGON

\author{
UNIVERSITY OF SOUTHERN CALIFORNIA \\ STANFORD UNIVERSITY \\ UNIVERSITY OF HAWAII \\ UNIVERSITY OF TOKYO \\ UNIVERSITY OF UTAH \\ WASHINGTON STATE UNIVERSITY \\ UNIVERSITY OF · WASHINGTON
}

The Supporting Institutions listed above contribute to the cost of publication of this Journal, but they are not owners or publishers and have no responsibility for its content or policies.

Mathematical papers intended for publication in the Pacific Journal of Mathematics should be in typed form or offset-reproduced, (not dittoed), double spaced with large margins. Please do not use built up fractions in the text of the manuscript. However, you may use them in the displayed equations. Underline Greek letters in red, German in green, and script in blue. The first paragraph or two must be capable of being used separately as a synopsis of the entire paper. Please propose a heading for the odd numbered pages of less than 35 characters. Manuscripts, in triplicate, may be sent to any one of the editors. Please classify according to the scheme of Math. Reviews, Index to Vol. 39. Supply name and address of author to whom proofs should be sent. All other communications should be addressed to the managing editor, or Elaine Barth, University of California, Los Angeles, California, 90024.

50 reprints to each author are provided free for each article, only if page charges have been substantially paid. Additional copies may be obtained at cost in multiples of 50 .

The Pacific Journal of Mathematics is issued monthly as of January 1966. Regular subscription rate: $\$ 84.00$ a year (6 Vols., 12 issues). Special rate: $\$ 42.00$ a year to individual members of supporting institutions.

Subscriptions, orders for numbers issued in the last three calendar years, and changes of address should be sent to Pacific Journal of Mathematics, P.O. Box 969, Carmel Valley, CA 93924, U.S.A. Older back numbers obtainable from Kraus Periodicals Co., Route 100, Millwood, NY 10546.

PUBLISHED BY PACIFIC JOURNAL OF MATHEMATICS, A NON-PROFIT CORPORATION

Printed at Kokusai Bunken Insatsusha (International Academic Printing Co., Ltd.). 8-8, 3-chome, Takadanobaba, Shinjuku-ku, Tokyo 160, Japan.

Copyright (C) 1979 by Pacific Journal of Mathematics Manufactured and first issued in Japan 


\section{Pacific Journal of Mathematics}

\section{Vol. 84, No. $2 \quad$ June, 1979}

Somesh Chandra Bagchi and Alladi Sitaram, Spherical mean periodic

functions on semisimple Lie groups ........................ 241

Billy Joe Ball, Quasicompactifications and shape theory............. 251

Maureen A. Bardwell, The o-primitive components of a regular ordered permutation group ................................ 261

Peter W. Bates and James R. Ward, Periodic solutions of higher order

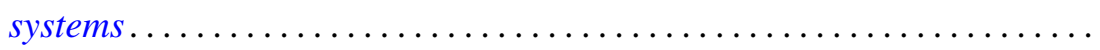

Jeroen Bruijning, A characterization of dimension of topological spaces by totally bounded pseudometrics......................... 283

Thomas Farmer, On the reduction of certain degenerate principal series

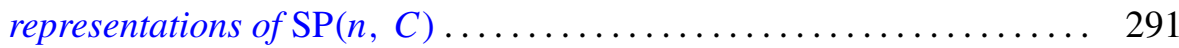

Richard P. Jerrard and Mark D. Meyerson, Homotopy with m-functions . . . . 305

James Edgar Keesling and Sibe Mardesic, A shape fibration with fibers of

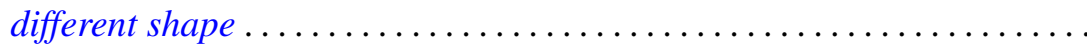

Guy Loupias, Cohomology over Banach crossed products. Application to bounded derivations and crossed homomorphisms ...............

Rainer Löwen, Symmetric planes ........................ 367

Alan L. T. Paterson, Amenable groups for which every topological left

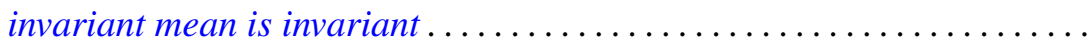

Jack Ray Porter and R. Grant Woods, Ultra-Hausdorff H-closed extensions

Calvin R. Putnam, Operators satisfying a $G_{1}$ condition .

Melvin Gordon Rothenberg and Jonathan David Sondow, Nonlinear smooth representations of compact Lie groups..............

Werner Rupp, Riesz-presentation of additive and $\sigma$-additive set-valued measures.

A. M. Russell, A commutative Banach algebra of functions of generalized variation

Judith D. Sally, Superregular sequences

Patrick Shanahan, On the signature of Grassmannians . . 